\title{
Synthesis and Application of a Fluorous Lawesson's Reagent: Convenient Chromatography-Free Product Purification
}

\author{
Zoltán Kaleta, ${ }^{\mathrm{a}}$ Gábor Tárkányi, ${ }^{\mathrm{b}}$ Ágnes Gömöry, ${ }^{\mathrm{b}}$ Ferenc Kálmán, ${ }^{\mathrm{c}}$ Tamás Nagy, ${ }^{\mathrm{c}}$ Tibor Soós ${ }^{*}$,a \\ ${ }^{a}$ Institute of Biomolecular Chemistry, Chemical Research Center of Hungarian \\ Academy of Science, P.O. Box 17, 1525 Budapest, Hungary \\ ${ }^{b}$ Institute of Structural Chemistry, Chemical Research Center of Hungarian Academy \\ of Science, P.O. Box 17, 1525 Budapest, Hungary \\ ${ }^{c}$ ComGenex Inc., 7 Záhony, 1031 Budapest, Hungary
}

General Information: All structures were verified by one- and two-dimensional NMR experiments. NMR spectra were recorded on a $400 \mathrm{MHz}$ (for ${ }^{1} \mathrm{H}$ ) Varian INOVA spectrometer using a Varian $5 \mathrm{~mm}$ ${ }^{1} \mathrm{H}-{ }^{19} \mathrm{~F} /\left\{{ }^{15} \mathrm{~N}-{ }^{31} \mathrm{P}\right\}$ Z-gradient indirect detection probe as well as a direct detection Varian ${ }^{15} \mathrm{~N}-{ }^{31} \mathrm{P} /\left\{{ }^{1} \mathrm{H}-\right.$ ${ }^{19} \mathrm{~F}$ \} switchable broadband probe. ${ }^{1} \mathrm{H}$ and ${ }^{13} \mathrm{C}$ chemical shifts are referenced to the residual solvent signals. Fluorine shifts are given relative to $\mathrm{CFCl}_{3}\left(\delta_{\mathrm{CFCl} 3}=0.0 \mathrm{ppm}\right) .{ }^{31} \mathrm{P}$ chemical shifts are referenced to the external reference: $85 \% \mathrm{H}_{3} \mathrm{PO}_{4}$ in capillary. For ${ }^{31} \mathrm{P}$ decoupled ${ }^{1} \mathrm{H}-\mathrm{NMR}$ spectra the WURST adiabatic heteronuclear decoupling scheme was used. ${ }^{1}$ Infrared spectra were recorded on a Thermo Nicolet Avatar 320 FT-IR spectrometer and are reported in frequency in wavenumbers. Mass spectra and high resolution mass spectra were obtained on VG ZAB2-SEQ tandem mass spectrometer. Melting points were determined by a Büchi apparatus and are uncorrected. All weights for small scale reactions were measured on a Mettler Toledo AX 105 DeltaRange®. All microwave experiments were conducted in Prolabo Synthewave S402 with constant 600W irradiation.

2-Methoxybenzaldehyde, anisaldehyde, 2,4-dimethoxybenzaldehyde, 2-methoxycinnamaldehyde, 10\% $\mathrm{Pd} / \mathrm{C}$ were obtained from Aldich and used as received. The $99.95 \%$ quality perdeuterated solvents were purchased from Cambridge Isotope Laboratories. Fluorous reverse phase silica gel (FluoroFlash ${ }^{\mathrm{TM}} \mathrm{SPE}$ cartridge 2 grams, $8 \mathrm{cc}$ tube) was purchased from Fluorous Technologies Inc.. After fluorous solid phase extractions the neutral alumine was discarded and the cartridges were regenerated by washing subsequently with acetone, THF methanol and THF. THF was distilled from sodium/benzophenone prior to use. 


\section{1-[(1E/Z)-4,4,5,5,6,6,7,7,8,8,9,9,10,10,11,11,11-heptadecafluoroundec-1-en-1-yl]-2-}

methoxybenzene (I)

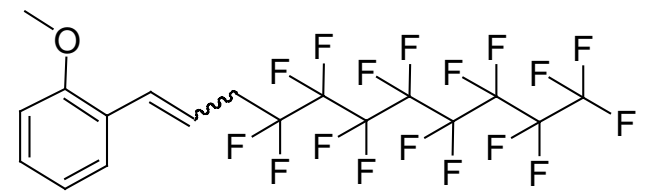

A $250 \mathrm{~mL}$ round bottom flask was charged with 2-methoxybenzaldehyde $(1.360 \mathrm{~g}, 10.0 \mathrm{mmol})$, $\mathrm{R}_{\mathrm{f} 8} \mathrm{CH}_{2} \mathrm{CH}_{2} \mathrm{PPh}_{3} \mathrm{I}^{2}(5,9.00 \mathrm{~g}, 10.8 \mathrm{mmol}), \mathrm{K}_{2} \mathrm{CO}_{3}(1.93 \mathrm{~g}, 14.0 \mathrm{mmol})$ and $200 \mathrm{~mL}$ of dioxane under an atmosphere of nitrogen. After establishing vigorous stirring, $\mathrm{H}_{2} \mathrm{O}(0.8 \mathrm{~mL})$ was added via syringe and the flask was moved to $90{ }^{\circ} \mathrm{C}$ oil bath. After 12 hours the flask was cooled to near ambient temperature, volatiles were evaporated. The residue was taken up in $\mathrm{CHCl}_{3}$ and water, the phases were partitioned, and the aqueous layer was further extracted with $\mathrm{CHCl}_{3}$ two times. The combined organic layers were washed with water, dried over $\mathrm{Na}_{2} \mathrm{SO}_{4}$. and evaporated under reduced pressure. The residue was suspended in hot hexanes, and placed on a short plug of hexanes packed silica gel $(5 \times 5 \mathrm{~cm})$, and eluted with hexanes. This filtrate was concentrated in vacuo to provide $\mathbf{I}$ as a colorless oil (5.124 g, $91 \%)$.

(Z-isomer) ${ }^{1} \mathrm{H}-\mathrm{NMR}\left(\mathrm{CDCl}_{3}, 400 \mathrm{MHz}\right): 3.05$ (dt, $\left.J=18.6,7.1 \mathrm{~Hz}, 2 \mathrm{H}, \mathrm{CH}_{2}\right), 3.85$ (s, 3H, $\left.\mathrm{CH}_{3}\right), 5.81$ $(\mathrm{dt}, J=11.4,7.1 \mathrm{~Hz}, 1 \mathrm{H},=\mathrm{CH}), 6.89(\mathrm{~d}, J=11.4 \mathrm{~Hz}, 1 \mathrm{H},=\mathrm{CH}), 6.93(\mathrm{~d}, J=8.2 \mathrm{~Hz}, 1 \mathrm{H}, \mathrm{Ar}-\mathrm{H}), 6.95-$ $7.01(\mathrm{~m}, 1 \mathrm{H}, \mathrm{Ar}-\mathrm{H}), 7.18$ (d, $J=7.2 \mathrm{~Hz}, 1 \mathrm{H}, \mathrm{Ar}-\mathrm{H}), 7.28-7.34(\mathrm{~m}, 1 \mathrm{H}, \mathrm{Ar}-\mathrm{H}) .{ }^{13} \mathrm{C}-\mathrm{NMR}\left(\mathrm{CDCl}_{3}, 100\right.$ $\mathrm{MHz}) * 30.8\left(\mathrm{t},{ }^{2} J_{\mathrm{C}-\mathrm{F}}=22.1 \mathrm{~Hz}, \mathrm{CH}_{2}\right), 55.3\left(\mathrm{CH}_{3}\right), 110.7(\mathrm{CH}), 118.1\left(\mathrm{t},{ }^{3} J_{\mathrm{C}-\mathrm{F}}=4.2 \mathrm{~Hz}, \mathrm{CH}\right), 120.3$ (CH), $124.8(\mathrm{C}), 129.2(\mathrm{CH}), 129.5(\mathrm{CH}), 131.5(\mathrm{CH}), 157.1(\mathrm{C})$.

* CF carbons are not listed

IR $(\mathrm{KBr}) \mathrm{cm}^{-1} ; 1601,1491,1467,1206,1151,754$.

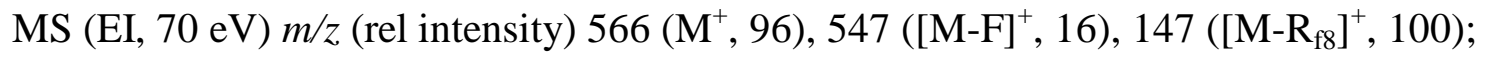

HRMS (EI, $70 \mathrm{eV}$ ) calcd. for $\left(\mathrm{C}_{18} \mathrm{H}_{11} \mathrm{~F}_{17} \mathrm{O}\right): 566.0538$, found: 566.0523. 


\section{1-(4,4,5,5,6,6,7,7,8,8,9,9,10,10,11,11,11-heptadecafluoroundecyl)-2-methoxybenzene (3a)}<smiles>COc1ccccc1CCCC(F)(F)C(F)(F)C(F)(F)C(F)(F)C(F)(F)C(F)(F)C(F)(F)C(F)(F)F</smiles>

A $500 \mathrm{~mL}$ round bottom flask was charged with $\mathbf{I}(2.830 \mathrm{~g}, 5.0 \mathrm{mmol}), 200 \mathrm{~mL}$ of ethanol, $100 \mathrm{~mL}$ of ethylacetate and $10 \% \mathrm{Pd} / \mathrm{C}(280 \mathrm{mg})$. The flask was evacuated and back-filled with $\mathrm{H}_{2}(3 \times, 1$ bar). The reaction mixture was stirred for $12 \mathrm{~h}$ at room temperarure. The flask was flushed with nitrogen and the reaction mixture was filtered over a short pad of Celite (ethyl acetate eluent) and the solvent was evaporated in vacuo to afford pure $3 \mathbf{a}$ as a colorless oil $(2.755 \mathrm{~g}, 97 \%)$.

${ }^{1} \mathrm{H}-\mathrm{NMR}\left(\mathrm{CDCl}_{3}, 400 \mathrm{MHz}\right): 1.89-2.01\left(\mathrm{~m}, 2 \mathrm{H}, \mathrm{CH}_{2}\right), 2.05-2.21\left(\mathrm{~m}, 2 \mathrm{H}, \mathrm{CH}_{2}\right), 2.75$ (t, J= 7.6 Hz, 2H, $\left.\mathrm{CH}_{2}\right), 3.86\left(\mathrm{~s}, 3 \mathrm{H}, \mathrm{CH}_{3}\right), 6.90(\mathrm{~d}, J=8.2 \mathrm{~Hz}, 1 \mathrm{H}, \mathrm{Ar}-\mathrm{H}), 6.91-6.96(\mathrm{~m}, 1 \mathrm{H}, \mathrm{Ar}-\mathrm{H}), 7.16$ (dd, J = 7.5, 1.4 $\mathrm{Hz}, 1 \mathrm{H}, \mathrm{Ar}-\mathrm{H}), 7.22-7.28$ (m, 1H, Ar-H). ${ }^{13} \mathrm{C}-\mathrm{NMR}(\mathrm{CDCl} 3,100 \mathrm{MHz}) *: 20.4\left(\mathrm{t},{ }^{3} J_{\mathrm{C}-\mathrm{F}}=3.4 \mathrm{~Hz}, \mathrm{CH}_{2}\right)$, $29.7\left(\mathrm{CH}_{2}\right), 30.6\left(\mathrm{t},{ }^{2} J_{\mathrm{C}-\mathrm{F}}=21.9 \mathrm{~Hz}, \mathrm{CH}_{2}\right), 55.1\left(\mathrm{CH}_{3}\right), 110.3(\mathrm{CH}), 120.5(\mathrm{CH}), 127.6(\mathrm{CH}), 129.2(\mathrm{C})$, $129.9(\mathrm{CH}), 157.5(\mathrm{C})$.

* CF carbons are not listed

IR (KBr) 2950, 1603, 1496, 1468, 1245, 1146, 1032, 753, $658 \mathrm{~cm}^{-1}$;

MS (EI, $70 \mathrm{eV}) \mathrm{m} / \mathrm{z}$ (rel intensity): $568\left(\mathrm{M}^{+}, 47\right), 549\left([\mathrm{M}-\mathrm{F}]^{+}, 11\right), 121\left(\left[\mathrm{M}-\mathrm{R}_{\mathrm{f} 8}-\mathrm{CH}_{2}-\mathrm{CH}_{2}\right]^{+}, 100\right)$

HRMS (EI, $70 \mathrm{eV}$ ) calcd. for $\left(\mathrm{C}_{18} \mathrm{H}_{13} \mathrm{~F}_{17} \mathrm{O}\right)$ : 568.0695, found: 568.0710.

\section{1-[1(E/Z)-4,4,5,5,6,6,7,7,8,8,9,9,10,10,11,11,11-heptadecafluoroundec-1-en-1-yl]-4-} methoxybenzene (II)

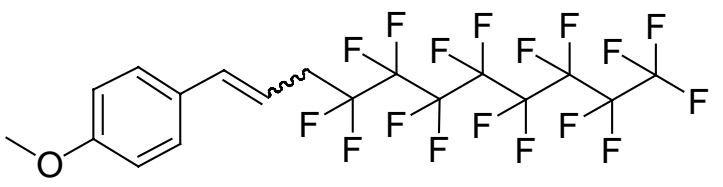


A $250 \mathrm{~mL}$ round bottom flask was charged with anisaldehyde (680 mg, $5.0 \mathrm{mmol}), \mathrm{R}_{\mathrm{f} 8} \mathrm{CH}_{2} \mathrm{CH}_{2} \mathrm{PPh}_{3} \mathrm{I}^{2}$ $(5,4.500 \mathrm{~g}, 5.4 \mathrm{mmol}), \mathrm{K}_{2} \mathrm{CO}_{3}(0.966 \mathrm{~g}, 7.0 \mathrm{mmol})$ and $130 \mathrm{~mL}$ of dioxane under an atmosphere of nitrogen. After establishing vigorous stirring, $\mathrm{H}_{2} \mathrm{O}(0.4 \mathrm{~mL})$ was added via syringe and the flask was moved to $90{ }^{\circ} \mathrm{C}$ oil bath. After 12 hours the flask was cooled to near ambient temperature, volatiles were evaporated. The residue was taken up in $\mathrm{CHCl}_{3}$ and water, the phases were partitioned, and the aqueous layer was further extracted with $\mathrm{CHCl}_{3}$ two times. The combined organic layers were washed with water, dried over $\mathrm{Na}_{2} \mathrm{SO}_{4}$. and evaporated under reduced pressure. The residue was suspended in hot hexanes, and placed on a short plug of hexanes packed silica gel $(1 \times 1 \mathrm{~cm})$, and eluted with hexanes. This filtrate was concentrated in vacuo to provide II as a colorless oil (1.650 g, $58 \%$ );

(Z-isomer) ${ }^{1} \mathrm{H}-\mathrm{NMR}\left(\mathrm{CDCl}_{3}, 400 \mathrm{MHz}\right): 3.12\left(\mathrm{dt}, J=18.4,6.9 \mathrm{~Hz}, 2 \mathrm{H}, \mathrm{CH}_{2}\right), 3.83\left(\mathrm{~s}, 3 \mathrm{H}, \mathrm{CH}_{3}\right), 5.63$ (dt, $J=11.4,7.1 \mathrm{~Hz}, 1 \mathrm{H},=\mathrm{CH}), 6.77(\mathrm{~d}, J=11.4 \mathrm{~Hz}, 1 \mathrm{H},=\mathrm{CH}), 6.90-6.95(\mathrm{~m}, 2 \mathrm{H}, \mathrm{Ar}-\mathrm{H}), 7.17-7.22$ (m, 2H, Ar-H). ${ }^{13} \mathrm{C}-\mathrm{NMR}\left(\mathrm{CDCl}_{3}, 100 \mathrm{MHz}\right) *: 30.6\left(\mathrm{t},{ }^{2} J_{\mathrm{C}-\mathrm{F}}=22.8 \mathrm{~Hz}, \mathrm{CH}_{2}\right), 55.2\left(\mathrm{CH}_{3}\right), 113.9(\mathrm{CH})$, $116.4\left(\mathrm{t},{ }^{3} J_{\mathrm{C}-\mathrm{F}}=4.8 \mathrm{~Hz}, \mathrm{CH}\right), 129.7(\mathrm{CH}), 134.9(\mathrm{CH}), 159.1(\mathrm{C})$.

* CF carbons are not listed

IR (KBr) 1610, 1513, 1243, 1207, 1177, 1151, $1114 \mathrm{~cm}^{-1}$;

MS (EI, $70 \mathrm{eV}) \mathrm{m} / \mathrm{z}$ (rel intensity) $566\left(\mathrm{M}^{+}, 88\right), 547\left([\mathrm{M}-\mathrm{F}]^{+}, 24\right), 147\left(\left[\mathrm{M}-\mathrm{R}_{\mathrm{f} 8}\right]^{+}, 100\right)$;

HRMS (EI, $70 \mathrm{eV}$ ) calcd. for $\left(\mathrm{C}_{18} \mathrm{H}_{11} \mathrm{~F}_{17} \mathrm{O}\right): 566.0538$, found: 566.0518.

\section{1-(4,4,5,5,6,6,7,7,8,8,9,9,10,10,11,11,11-heptadecafluoroundecyl)-4-methoxybenzene (3b)}

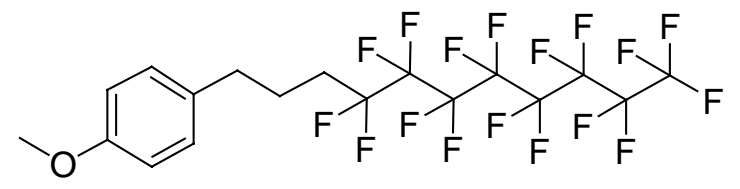

A $250 \mathrm{~mL}$ round bottom flask was charged with II (1415 mg, $2.5 \mathrm{mmol}), 100 \mathrm{~mL}$ of ethanol, $50 \mathrm{~mL}$ of ethylacetate and $10 \% \mathrm{Pd} / \mathrm{C}(140 \mathrm{mg})$. The flask was evacuated and back-filled with $\mathrm{H}_{2}(3 \times, 1$ bar). The reaction mixture was stirred for $12 \mathrm{~h}$ at room temperarure. The flask was flushed with nitrogen and the reaction mixture was filtered over a short pad of Celite (ethyl acetate eluent) and the solvent was evaporated in vacuo to afford pure $\mathbf{3 b}$ as a white solid (1392 mg, $98 \%$ ). mp: $36-37{ }^{\circ} \mathrm{C}$; 
${ }^{1} \mathrm{H}-\mathrm{NMR}\left(\mathrm{CDCl}_{3}, 400 \mathrm{MHz}\right): 1.89-1.99\left(\mathrm{~m}, 2 \mathrm{H}, \mathrm{CH}_{2}\right), 2.01-2.18\left(\mathrm{~m}, 2 \mathrm{H}, \mathrm{CH}_{2}\right), 2.65(\mathrm{t}, J=7.6 \mathrm{~Hz}, 2 \mathrm{H}$, $\mathrm{CH}_{2}$ ), 3.80 (s, 3H, CH 3 ), 6.83-6.91 (m, 2H, Ar-H), 7.10-7.16 (m, 2H, Ar-H). ${ }^{13} \mathrm{C}-\mathrm{NMR}(\mathrm{CDCl} 3,100$ $\mathrm{MHz})^{*}: 22.1\left(\mathrm{t},{ }^{3} J_{\mathrm{C}-\mathrm{F}}=3.6 \mathrm{~Hz}, \mathrm{CH}_{2}\right), 30.3\left(\mathrm{t},{ }^{2} J_{\mathrm{C}-\mathrm{F}}=22.4 \mathrm{~Hz}, \mathrm{CH}_{2}\right), 34.1\left(\mathrm{CH}_{2}\right), 55.2\left(\mathrm{CH}_{3}\right), 114.0$ $(\mathrm{CH}), 129.3(\mathrm{CH}), 132.7(\mathrm{C}), 158.1(\mathrm{C})$.

* CF carbons are not listed

IR (KBr) 2962, 1610, 1515, 1245, 1202, 1148, 1115, 1027, $664 \mathrm{~cm}^{-1}$;

HRMS (EI, $70 \mathrm{eV}$ ) calcd. for $\left(\mathrm{C}_{18} \mathrm{H}_{13} \mathrm{~F}_{17} \mathrm{O}\right): 568.0695$, found: 568.0698.

\section{1-[(1E/Z)-4,4,5,5,6,6,7,7,8,8,9,9,10,10,11,11,11-heptadecafluoroundec-1-en-1-yl]-2,4-} dimethoxybenzene (III)

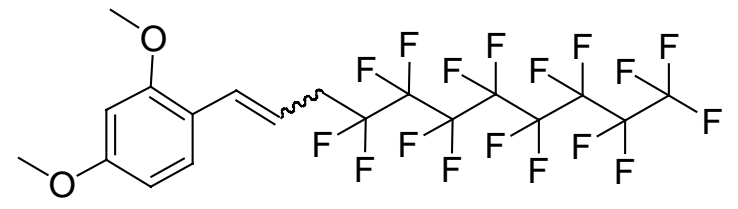

A $250 \mathrm{~mL}$ round bottom flask was charged with 2,4-dimethoxybenzaldehyde (830 $\mathrm{mg}, 5.0 \mathrm{mmol})$, $\mathrm{R}_{\mathrm{f} 8} \mathrm{CH}_{2} \mathrm{CH}_{2} \mathrm{PPh}_{3} \mathrm{I}^{2}(5,6.270 \mathrm{~g}, 7.5 \mathrm{mmol}), \mathrm{K}_{2} \mathrm{CO}_{3}(1.035 \mathrm{~g}, 7.5 \mathrm{mmol})$ and $130 \mathrm{~mL}$ of dioxane under an atmosphere of nitrogen. After establishing vigorous stirring, $\mathrm{H}_{2} \mathrm{O}(0.4 \mathrm{~mL})$ was added via syrenge and the flask was moved to $90{ }^{\circ} \mathrm{C}$ oil bath. After 3 days the flask was cooled to near ambient temperature, volatiles were evaporated. The residue was taken up in $\mathrm{CHCl}_{3}$ and water, the phases were partitioned, and the aqueous layer was further extracted with $\mathrm{CHCl}_{3}$ two times. The combined organic layers were washed with water, dried over $\mathrm{Na}_{2} \mathrm{SO}_{4}$. and evaporated under reduced pressure. The residue was suspended in hot hexanes, and placed on a short plug of hexanes packed silica gel $(5 \times 5 \mathrm{~cm})$, and eluted with hexanes. This filtrate was concentrated in vacuo to provide III as a colorless oil (2.740 g, $92 \%)$;

(Z-isomer) ${ }^{1} \mathrm{H}-\mathrm{NMR}\left(\mathrm{CDCl}_{3}, 400 \mathrm{MHz}\right): 3.04$ (dt, $\left.J=18.9,7.1 \mathrm{~Hz}, 2 \mathrm{H}, \mathrm{CH}_{2}\right), 3.82\left(\mathrm{~s}, 3 \mathrm{H}, \mathrm{CH}_{3}\right), 3.83$ $\left(\mathrm{s}, 3 \mathrm{H}, \mathrm{CH}_{3}\right), 5.72(\mathrm{dt}, J=11.3,7.2 \mathrm{~Hz}, 1 \mathrm{H},=\mathrm{CH}), 6.44-6.53(\mathrm{~m}, 2 \mathrm{H}, \mathrm{Ar}-\mathrm{H}), 6.81(\mathrm{~d}, J=11.3 \mathrm{~Hz}, 1 \mathrm{H}$, $=\mathrm{CH}), 7.09(\mathrm{~d}, J=9.3 \mathrm{~Hz}, 1 \mathrm{H}, \mathrm{Ar}-\mathrm{H}) .{ }^{13} \mathrm{C}-\mathrm{NMR}\left(\mathrm{CDCl}_{3}, 100 \mathrm{MHz}\right)^{*}: 30.8\left(\mathrm{t},{ }^{2} J_{\mathrm{C}-\mathrm{F}}=22.6 \mathrm{~Hz}, \mathrm{CH}_{2}\right)$, $55.3\left(\mathrm{CH}_{3}\right), 55.4\left(\mathrm{CH}_{3}\right), 98.6(\mathrm{CH}), 104.0(\mathrm{CH}), 116.9\left(\mathrm{t},{ }^{3} J_{\mathrm{C}-\mathrm{F}}=4.5 \mathrm{~Hz}, \mathrm{CH}\right), 117.6(\mathrm{C}) 129.9(\mathrm{CH})$, $131.0(\mathrm{CH}), 158.2(\mathrm{C}), 160.7(\mathrm{C})$.

* CF carbons are not listed 
IR $(\mathrm{KBr}) \mathrm{cm}^{-1} 1613,1506,1468,1210,1152,1038$.

MS (EI, $70 \mathrm{eV}) \mathrm{m} / z$ (rel intensity) $596\left(\mathrm{M}^{+}, 58\right), 577\left([\mathrm{M}-\mathrm{F}]^{+}, 14\right), 177\left(\left[\mathrm{M}-\mathrm{R}_{\mathrm{f} 8}\right]^{+}, 50\right), 151\left(\left[\mathrm{M}-\mathrm{R}_{\mathrm{f} 8}{ }^{-}\right.\right.$ $\left.\mathrm{CH}=\mathrm{CH}]^{+}, 100\right)$;

HRMS (EI, $70 \mathrm{eV})$ calcd. for $\left(\mathrm{C}_{19} \mathrm{H}_{13} \mathrm{~F}_{17} \mathrm{O}_{2}\right)$ : 596.0644, found: 596.0633.

\section{1-(4,4,5,5,6,6,7,7,8,8,9,9,10,10,11,11,11-heptadecafluoroundecyl)-2,4-dimethoxybenzene (3c)}

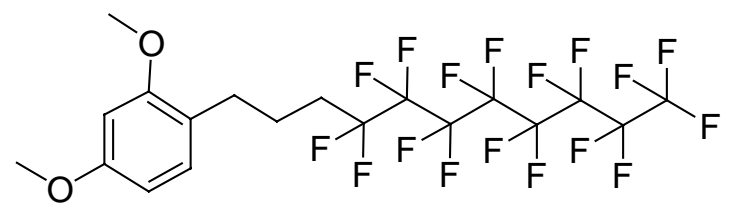

A $250 \mathrm{~mL}$ round bottom flask was charged with III $(1.788 \mathrm{~g}, 3.0 \mathrm{mmol}), 100 \mathrm{~mL}$ of ethanol, $50 \mathrm{~mL}$ of ethylacetate and $10 \% \mathrm{Pd} / \mathrm{C}(180 \mathrm{mg})$. The flask was evacuated and back-filled with $\mathrm{H}_{2}(3 \times, 1$ bar). The reaction mixture was stirred for $12 \mathrm{~h}$ at room temperarure. The flask was flushed with nitrogen and the reaction mixture was filtered over a short pad of Celite (ethyl acetate eluent) and the solvent was evaporated in vacuo to afford pure $3 \mathrm{c}$ as a white solid $(1.758 \mathrm{~g}, 98 \%)$. mp: $28-30{ }^{\circ} \mathrm{C}$;

${ }^{1} \mathrm{H}-\mathrm{NMR}\left(\mathrm{CDCl}_{3}, 400 \mathrm{MHz}\right): 1.84-1.95\left(\mathrm{~m}, 2 \mathrm{H}, \mathrm{CH}_{2}\right), 2.01-2.18\left(\mathrm{~m}, 2 \mathrm{H}, \mathrm{CH}_{2}\right), 2.66(\mathrm{t}, J=7.6 \mathrm{~Hz}, 2 \mathrm{H}$, $\left.\mathrm{CH}_{2}\right), 3.82\left(\mathrm{~s}, 6 \mathrm{H}, \mathrm{CH}_{3}\right), 6.46(\mathrm{dd}, J=8.0,2.1 \mathrm{~Hz}, 1 \mathrm{H}, \mathrm{Ar}-\mathrm{H}), 6.49$ (d, J=2.1 Hz, 1H, Ar-H), $7.03(\mathrm{~d}, J$ $=8.0 \mathrm{~Hz}, 1 \mathrm{H}, \mathrm{Ar}-\mathrm{H}) .{ }^{13} \mathrm{C}-\mathrm{NMR}\left(\mathrm{CDCl}_{3}, 100 \mathrm{MHz}\right)^{*}: 20.6\left(\mathrm{t},{ }^{3} J_{\mathrm{C}-\mathrm{F}}=3.4 \mathrm{~Hz}, \mathrm{CH}_{2}\right), 29.0\left(\mathrm{CH}_{2}\right), 30.5(\mathrm{t}$, $\left.{ }^{2} J_{\mathrm{C}-\mathrm{F}}=22.5 \mathrm{~Hz}, \mathrm{CH}_{2}\right), 55.1\left(\mathrm{CH}_{3}\right), 55.3\left(\mathrm{CH}_{3}\right), 98.6(\mathrm{CH}), 103.9(\mathrm{CH}), 121.5(\mathrm{C}), 130.1(\mathrm{CH}), 158.4$ (C), $159.6(\mathrm{C})$.

* CF carbons are not listed

IR (KBr) 2962, 1512, 1245, 1202, 1148, 1115, 1027, $664 \mathrm{~cm}^{-1}$;

MS (EI, $70 \mathrm{eV}) \mathrm{m} / z$ (rel intensity) $598\left(\mathrm{M}^{+}, 52\right), 579\left([\mathrm{M}-\mathrm{F}]^{+}, 21\right), 151\left(\left[\mathrm{M}-\mathrm{R}_{\mathrm{f} 8}-\mathrm{CH}_{2}-\mathrm{CH}_{2}\right]^{+}, 100\right), 121$ $\left(\left[\mathrm{M}-\mathrm{R}_{\mathrm{f8}}-58\right]^{+}, 22\right)$;

HRMS (EI, $70 \mathrm{eV})$ calcd. for $\left(\mathrm{C}_{19} \mathrm{H}_{15} \mathrm{~F}_{17} \mathrm{O}_{2}\right)$ : 598.0801, found: 598.0829. 


\section{1-(6,6,7,7,8,8,9,9,10,10,11,11,12,12,13,13,13-heptadecafluorotrideca-1,3-dien)-1-yl-2-}

methoxybenzene (6)

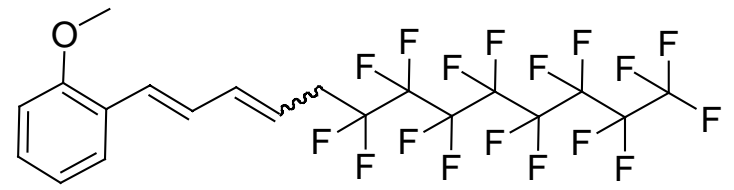

A $1 \mathrm{~L}$ round bottom flask was charged with 2-methoxycinnamaldehyde $(4,3.240 \mathrm{~g}, 20.0 \mathrm{mmol})$, $\mathrm{R}_{\mathrm{f8}} \mathrm{CH}_{2} \mathrm{CH}_{2} \mathrm{PPh}_{3} \mathrm{I}^{2}(5,20.90 \mathrm{~g}, 25.0 \mathrm{mmol}), \mathrm{K}_{2} \mathrm{CO}_{3}(5.520 \mathrm{~g}, 40.0 \mathrm{mmol})$ and $500 \mathrm{~mL}$ of dioxane under an atmosphere of nitrogen. After establishing vigorous stirring, $\mathrm{H}_{2} \mathrm{O}(5.0 \mathrm{~mL})$ was added via syrenge and the flask was moved to $90{ }^{\circ} \mathrm{C}$ oil bath. After 12 hours the flask was cooled to near ambient temperature, volatiles were evaporated. The residue was taken up in $\mathrm{CHCl}_{3}$ and water, the phases were partitioned, and the aqueous layer was further extracted with $\mathrm{CHCl}_{3}$ two times. The combined organic layers were washed with water, dried over $\mathrm{Na}_{2} \mathrm{SO}_{4}$. and evaporated under reduced pressure. The residue was suspended in hot hexanes, and placed on a short plug of hexanes packed silica gel $(6 \times 6 \mathrm{~cm})$, and eluted with hexanes. This filtrate was concentrated in vacuo to provide 6 as a white solid (10.250 g, 86\%) mp: $81-82^{\circ} \mathrm{C}$;

(E,Z-isomer) ${ }^{1} \mathrm{H}-\mathrm{NMR}\left(\mathrm{CDCl}_{3}, 400 \mathrm{MHz}\right): 3.12\left(\mathrm{dt}, J=18.6,8.3 \mathrm{~Hz}, 2 \mathrm{H}, \mathrm{CH}_{2}\right), 3.88\left(\mathrm{~s}, 3 \mathrm{H}, \mathrm{CH}_{3}\right)$, $5.50(\mathrm{dt}, J=10.6,7.6 \mathrm{~Hz}, 1 \mathrm{H},=\mathrm{CH}), 6.51-6.59(\mathrm{~m}, 1 \mathrm{H},=\mathrm{CH}), 6.91(\mathrm{~d}, J=8.3 \mathrm{~Hz}, 1 \mathrm{H}, \mathrm{Ar}-\mathrm{H}), 6.95-$ $7.01(\mathrm{~m}, 1 \mathrm{H}, \mathrm{Ar}-\mathrm{H}), 7.02-7.06(\mathrm{~m}, 2 \mathrm{H},=\mathrm{CH}), 7.25-7.31(\mathrm{~m}, 1 \mathrm{H}, \mathrm{Ar}-\mathrm{H}), 7.52(\mathrm{~d}, J=7.8 \mathrm{~Hz}, 1 \mathrm{H}, \mathrm{Ar}-\mathrm{H})$. ${ }^{13} \mathrm{C}-\mathrm{NMR}\left(\mathrm{CDCl}_{3}, 100 \mathrm{MHz}\right) *: 30.1\left(\mathrm{t},{ }^{2} J_{\mathrm{C}-\mathrm{F}}=23.4 \mathrm{~Hz}, \mathrm{CH}_{2}\right), 55.3\left(\mathrm{CH}_{3}\right), 110.9(\mathrm{CH}), 116.0\left(\mathrm{t},{ }^{3} J_{\mathrm{C}-\mathrm{F}}=\right.$ $4.1 \mathrm{~Hz}, \mathrm{CH}), 120.7(\mathrm{CH}), 123.3(\mathrm{CH}), 125.9(\mathrm{C}), 127.0(\mathrm{CH}), 129.2(\mathrm{CH}), 130.7(\mathrm{CH}), 135.7(\mathrm{C}), 157.2$ (C).

* CF carbons are not listed

IR (KBr) 1597, 1491, 1251, 1202, 1148, 752, $657 \mathrm{~cm}^{-1}$;

MS (EI, $70 \mathrm{eV}) \mathrm{m} / z$ (rel intensity) $592\left(\mathrm{M}^{+}, 94\right), 573\left([\mathrm{M}-\mathrm{F}]^{+}, 10\right), 173\left(\left[\mathrm{M}-\mathrm{R}_{\mathrm{f8}}\right]^{+}, 40\right), 159\left(\left[\mathrm{M}-\mathrm{R}_{\mathrm{f} 8}{ }^{-}\right.\right.$ $\left.\left.\mathrm{CH}_{2}\right]^{+}, 100\right), 144\left([173-29]^{+}, 13\right)$;

HRMS (EI, $70 \mathrm{eV}$ ) calcd. for $\left(\mathrm{C}_{20} \mathrm{H}_{13} \mathrm{~F}_{17} \mathrm{O}\right)$ : 592.0695, found: 592.0672. 


\section{1-(6,6,7,7,8,8,9,9,10,10,11,11,12,12,13,13,13-heptadecafluorotridecyl)-2-methoxybenzene (7).}

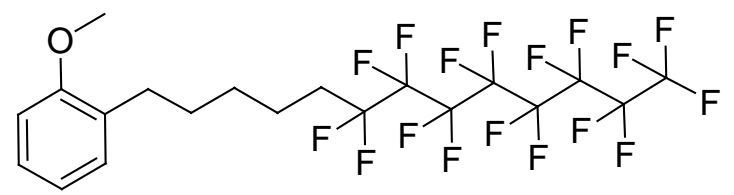

A 1.0 L autoclave was charged with $6(9.500 \mathrm{~g}, 16.0 \mathrm{mmol}) 300 \mathrm{~mL}$ of ethanol, $200 \mathrm{~mL}$ of ethylacetate and $10 \% \mathrm{Pd} / \mathrm{C}(950 \mathrm{mg})$. The autoclave was evacuated and back-filled with $\mathrm{H}_{2}(3 \times, 5$ bar $)$. The reaction mixture was stirred for $12 \mathrm{~h}$ at room temperarure. The flask was flushed with nitrogen and the reaction mixture was filtered over a short pad of Celite (ethyl acetate eluent). After removal of volatiles the crude product was bulb-to-bulb distilled $\left(200{ }^{\circ} \mathrm{C}\right.$ at $2.1 * 10^{-1}$ mbar) in a Kugelrohr apparatus to yield pure 7 as a white solid (9.046 g, 95\%). mp: $29-30{ }^{\circ} \mathrm{C}$;

${ }^{1} \mathrm{H}-\mathrm{NMR}\left(\mathrm{CDCl}_{3}, 400 \mathrm{MHz}\right): 1.40-1.50\left(\mathrm{~m}, 2 \mathrm{H}, \mathrm{CH}_{2}\right), 1.59-1.72\left(\mathrm{~m}, 4 \mathrm{H}, \mathrm{CH}_{2}\right), 1.98-2.16(\mathrm{~m}, 2 \mathrm{H}$, $\left.\mathrm{CH}_{2}\right), 2.61-2.68\left(\mathrm{~m}, 2 \mathrm{H}, \mathrm{CH}_{2}\right), 3.84\left(\mathrm{~s}, 3 \mathrm{H}, \mathrm{CH}_{3}\right), 6.86(\mathrm{~d}, J=8.5 \mathrm{~Hz}, 1 \mathrm{H}, \mathrm{Ar}-\mathrm{H}), 6.88-6.93$ (m, 1H, Ar$\mathrm{H}), 7.13(\mathrm{dd}, J=7.5,1.4 \mathrm{~Hz}, 1 \mathrm{H}, \mathrm{Ar}-\mathrm{H}), 7.17-7.23$ (m, 1H, Ar-H). ${ }^{13} \mathrm{C}-\mathrm{NMR}\left(\mathrm{CDCl}_{3}, 100 \mathrm{MHz}\right) *: 19.9$ $\left(\mathrm{t},{ }^{3} J_{\mathrm{C}-\mathrm{F}}=3.9 \mathrm{~Hz}, \mathrm{CH}_{2}\right), 28.8\left(\mathrm{CH}_{2}\right), 29.4\left(\mathrm{CH}_{2}\right), 29.8\left(\mathrm{CH}_{2}\right), 30.8\left(\mathrm{t},{ }^{2} J_{\mathrm{C}-\mathrm{F}}=22.5 \mathrm{~Hz}, \mathrm{CH}_{2}\right), 55.2\left(\mathrm{CH}_{3}\right)$, $110.2(\mathrm{CH}), 120.4(\mathrm{CH}), 127.0(\mathrm{CH}), 129.8(\mathrm{CH}), 130.6(\mathrm{C}), 157.4(\mathrm{C})$.

* CF carbons are not listed

IR (KBr) 1497, 1471, 1245, 1209, 1146, 1038, 755, $659 \mathrm{~cm}^{-1}$;

MS (EI, $70 \mathrm{eV}) \mathrm{m} / z$ (rel intensity) $596\left(\mathrm{M}^{+}, 37\right), 577\left([\mathrm{M}-\mathrm{F}]^{+}, 10\right), 122\left(\left[\mathrm{M}-\mathrm{R}_{\mathrm{f8}}-\left(\mathrm{CH}_{2}\right)_{4}\right]^{+}, 100\right), 91$ $\left([122-30]^{+}, 31\right)$;

HRMS (EI, $70 \mathrm{eV}$ ) calcd. for $\left(\mathrm{C}_{20} \mathrm{H}_{17} \mathrm{~F}_{17} \mathrm{O}\right)$ : 596.1008, found: 596.1007. 


\section{2,4-bis[3-(6,6,7,7,8,8,9,9,10,10,11,11,12,12,13,13,13-heptadecafluorotridecyl)-4-methoxyphenyl]-}

\section{1,3,2,4-dithiadiphosphetane 2,4-disulfide (8).}

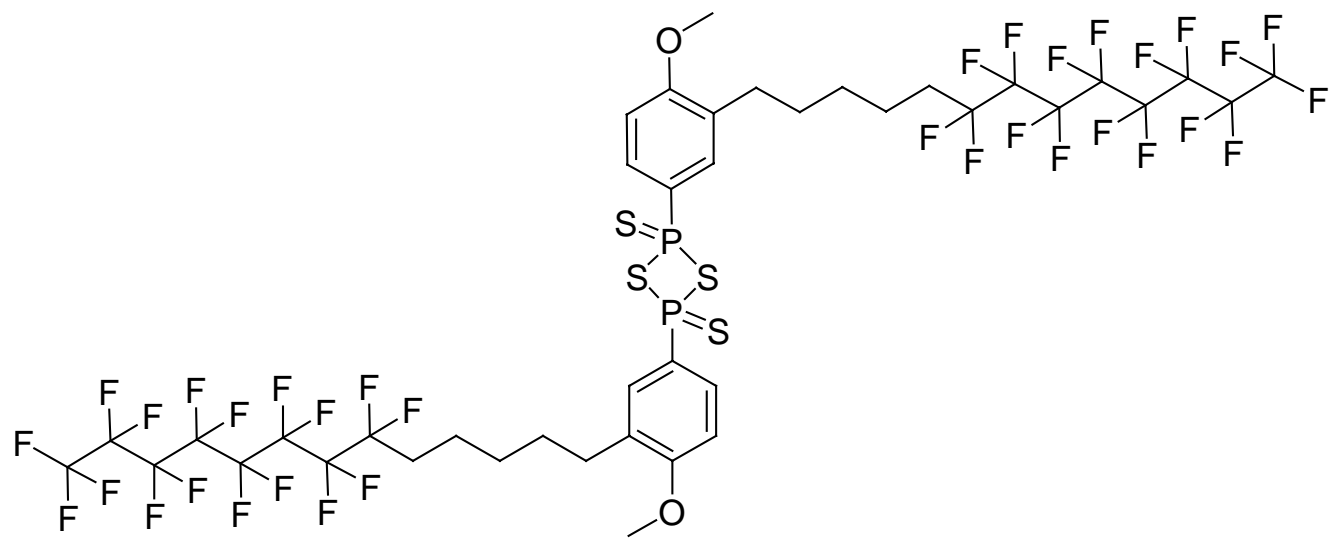

A $50 \mathrm{~mL}$ round bottom flask was charged with $7(8.940 \mathrm{~g}, 15.0 \mathrm{mmol})$, phosphorous pentasulfide (2, $341 \mathrm{mg}, 1.5 \mathrm{mmol}$ ), and $10 \mathrm{~mL}$ of dry 1,2-dichlorobenzene under an atmosphere of argon. The reaction mixture was stirred at $150{ }^{\circ} \mathrm{C}$ for 4 hours then cooled to ambient temperature. The resulted precipitate was filtered off and washed with anhydrous hexanes to yield $\mathbf{8}$ as pale yellow solid (937 $\mathrm{mg}, 0.68$ mmol, 45\%). mp: 115-118 (dec.). The filtrate was concentrated in vacuo and bulb-to-bulb distilled (200 ${ }^{\circ} \mathrm{C}$ at $\left.2.1^{*} 10^{-1} \mathrm{mbar}\right)$ in a Kugelrohr apparatus to recover the starting material $7(6.711 \mathrm{~g}, 82 \%$ efficiency ).

The NMR characterization of fluorous Lawesson's reagent $(\mathbf{8})$ is rather difficult due to its low solubility in organic solvents (solubility $\leq$ ca. $3 \mathrm{mM}$ ) and the high tendency to hydrolyze. When $\mathbf{8}$ was dissolved into THF- $\mathrm{d}_{8}$ a single component (probably a monomer) was observed (Figure S18). Its ${ }^{31} \mathrm{P}$ shift in $\mathrm{C}_{6} \mathrm{D}_{6}$ was $17.5 \mathrm{ppm}$. (Figure S17) which is consistent with literature data, however, in THF-d $\mathrm{d}_{8}$ different shift

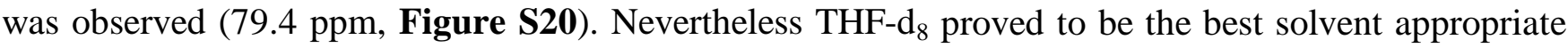
for reproducible characterization of $\mathbf{8}$ with respect to sample purity. Additional we note that at room temperature it was not possible to run direct ${ }^{13} \mathrm{C}$ experiments due to the low solubility. However, we gained further structural details by two dimensional proton detected heterocorrelated spectra ${ }^{1} \mathrm{H}-{ }^{13} \mathrm{C}$ HSQC, ${ }^{1} \mathrm{H}-{ }^{13} \mathrm{C}-\mathrm{HMQC}$ and ${ }^{1} \mathrm{H}$-DOSY NMR. With the latter method aliphatic and aromatic protons were correlated in the diffusion dimension. The DOSY experiment was carried out at $25^{\circ} \mathrm{C}$ in a $2 \mathrm{~mm}$ capillary inserted in a $3 \mathrm{~mm}$ NMR tube to minimize convection effects. Performa I. gradient amplifier 
(max. 20 gauss $/ \mathrm{cm}$ ) was used. The gradient strength was calibrated by using $5 \mathrm{w} / \mathrm{w} \%$ sucrose in $\mathrm{D}_{2} \mathrm{O}$ at $25{ }^{\circ} \mathrm{C}\left(D=5.22 \mathrm{E}^{-10} \mathrm{~m}^{2} / \mathrm{s}\right)$. The bipolar pulse-pair stimulated echo (Dbppste) sequence was used for acquiring diffusion data with $50 \mathrm{~ms}$ diffusion delay, 16 squared increments for gradient levels and 64 transients. The Varian DOSY package was used for the processing.

${ }^{1} \mathrm{H}-\mathrm{NMR}\left(\mathrm{THF}-\mathrm{d}_{8}, 400 \mathrm{MHz}, 3 \mathrm{mM}\right)$ : 1.43-1.52 (m, 2H, $\left.\mathrm{CH}_{2}\right), 1.60-1.70\left(\mathrm{~m}, 4 \mathrm{H}, \mathrm{CH}_{2}\right), 2.10-2.27(\mathrm{~m}$, $\left.2 \mathrm{H}, \mathrm{CH}_{2}\right), 2.64-2.72\left(\mathrm{~m}, 2 \mathrm{H}, \mathrm{CH}_{2}\right), 3.87\left(\mathrm{~s}, 3 \mathrm{H}, \mathrm{CH}_{3}\right), 6.99\left(\mathrm{dd}, \mathrm{J}_{\mathrm{H}-\mathrm{H}}=8.4 \mathrm{~Hz}, \mathrm{~J}_{\mathrm{H}-\mathrm{P}}=3.6,1 \mathrm{H}, \mathrm{Ar}-\mathrm{H}\right), 7.76$ $\left(\mathrm{dd}, \mathrm{J}_{\mathrm{H}-\mathrm{H}}=1.9 \mathrm{~Hz}, \mathrm{~J}_{\mathrm{H}-\mathrm{P}}=15.1,1 \mathrm{H}, \mathrm{Ar}-\mathrm{H}\right), 8.73\left(\mathrm{ddd}, \mathrm{J}_{\mathrm{H}-\mathrm{H}}=8.4, \mathrm{~J}_{\mathrm{H}-\mathrm{H}}=1.9 \mathrm{~Hz}, \mathrm{~J}_{\mathrm{H}-\mathrm{P}}=14.9,1 \mathrm{H}, \operatorname{Ar}-\mathrm{H}\right) .{ }^{13} \mathrm{C}-$ NMR (THF-d 8,100 MHz, HSQC/HMQC)*: $21.0\left(\mathrm{CH}_{2}\right), 29.7\left(\mathrm{CH}_{2}\right), 30.4\left(\mathrm{CH}_{2}\right), 30.9\left(\mathrm{CH}_{2}\right), 31.5$ $\left(\mathrm{CH}_{2}\right), 55.9\left(\mathrm{CH}_{3}\right), 110.4(\mathrm{CH}), 130.7,\left(\mathrm{~d}, J_{\mathrm{C}-\mathrm{P}}=28.5 \mathrm{~Hz}, \mathrm{CH}\right) 131.2(\mathrm{C}), 132.4(\mathrm{CH}), 161.4(\mathrm{C}) .{ }^{19} \mathrm{~F}-$ NMR (THF-d $8,376 \mathrm{MHz}):-81.4(\mathrm{t}, J=10.0 \mathrm{~Hz}, 3 \mathrm{~F}),-114.7$ (m, 2F), -122.3 (m, 2F), -122.4 (m, 4F), $123.3(\mathrm{~m}, 2 \mathrm{~F}),-124.0(\mathrm{~m}, 2 \mathrm{~F}),-126.7(\mathrm{~m}, 2 \mathrm{~F}) .{ }^{31} \mathrm{P}-\mathrm{NMR}\left(\mathrm{THF}-\mathrm{d}_{8}, 30^{\circ} \mathrm{C}, 162 \mathrm{MHz}\right): 79.4\left(\mathrm{t}, J_{\mathrm{H}-\mathrm{P}}=15.0\right.$ $\mathrm{Hz}, 1 \mathrm{P})$.

* CF carbons are not listed

IR (KBr) 2937, 2859, 2853, 1588, 1489, 1255, 1203, 1150, 1111, 1032, 818, 681, $624 \mathrm{~cm}^{-1}$;

MS (EI, $70 \mathrm{eV}) \mathrm{m} / z$ (rel intensity) $690\left([\mathrm{M} / 2]^{+}, 100\right), 627\left(\{[\mathrm{M} / 2]-\mathrm{PS}\}^{+}, 76\right), 215\left(\left\{[\mathrm{M} / 2]-\mathrm{R}_{\mathrm{f8}}-\left(\mathrm{CH}_{2}\right)_{5}\right\}^{+}\right.$, 41), $152\left([215-\mathrm{PS}]^{+}, 17\right)$;

HRMS (EI, $70 \mathrm{eV}$ ) monomer calcd. for $\left(\mathrm{C}_{20} \mathrm{H}_{16} \mathrm{~F}_{17} \mathrm{OPS}_{2}\right)$ : 690.0109, found: 690.0136; (monomer-PS) calcd. for $\left(\mathrm{C}_{20} \mathrm{H}_{16} \mathrm{~F}_{17} \mathrm{OS}\right)$ : 627.0650, found: 627.0642 .

\section{General procedures for use of fluorous Lawesson's reagent (8), thermal thionation reactions.}

Under an argon atmosphere a Schlenk tube was charged with the corresponding amide 9a-g (0.2 mmol), fluorous Lawesson's reagent $8(138 \mathrm{mg}, 0.1 \mathrm{mmol})$ and $4 \mathrm{~mL}$ of anhydrous THF. After the reaction was complete $2.0 \mathrm{~g}$ of neutral aluminium oxide (Brockmann II, neutral) was added and the solvent was evaporated to dryness. The resulting solid was placed on a short column packed with FluoroFlash $^{\mathrm{TM}}$ SPE silica $(2.0 \mathrm{~g})$. The column was eluted with methanol/water $8 / 2$ and evaporated to dryness to give the pure product 10a-g.

\section{N,N-Dimethylthioacetamide (10a) ${ }^{4}$}


$\|_{N^{\prime}}^{S}$

The reaction was carried out for $1.5 \mathrm{~h}$ according to the general thermal thionation procedure.

${ }^{1} \mathrm{H}-\mathrm{NMR}:\left(\mathrm{CDCl}_{3}, 200 \mathrm{MHz}\right) \delta 3.48\left(\mathrm{~s}, 3 \mathrm{H}, \mathrm{N}-\mathrm{CH}_{3}\right), 3.29$ (s, 3H, N-CH$\left.)_{3}\right) 2.64\left(\mathrm{~s}, 3 \mathrm{H}, \mathrm{CH}_{3}\right) ; \mathrm{IR}(\mathrm{KBr})$ 2932, 1535, 1420, 1390, 1362, 1278, 1180, 1124, 1015, 864, $656 \mathrm{~cm}^{-1}$; MS (EI, $\left.70 \mathrm{eV}\right) \mathrm{m} / z($ rel intensity) $103\left(\mathrm{M}^{+}, 100\right), 88\left(\left[\mathrm{M}-\mathrm{CH}_{3}\right]^{+}, 9\right), 70\left([\mathrm{M}-\mathrm{SH}]^{+}, 27\right), 59\left(\left[\mathrm{CH}_{3} \mathrm{CS}\right]^{+}, 36\right), 44\left(\left[\mathrm{~N}\left(\mathrm{CH}_{3}\right)_{2}\right]^{+}, 30\right)$.

\section{N-methylpyrrolidine-2-thione (10b) ${ }^{5}$}

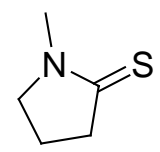

The reaction was carried out for $2 \mathrm{~h}$ according to the general thermal thionation procedure.

${ }^{1} \mathrm{H}-\mathrm{NMR}:\left(\mathrm{CDCl}_{3}, 200 \mathrm{MHz}\right) \delta 3.72\left(\mathrm{t}, J=7.2 \mathrm{~Hz}, 2 \mathrm{H}, \mathrm{CH}_{2}\right), 3.24\left(\mathrm{~s}, 3 \mathrm{H}, \mathrm{N}-\mathrm{CH}_{3}\right), 3.02\left(\mathrm{~m}, 2 \mathrm{H}, \mathrm{CH}_{2}\right)$, 2.13-1.96 (m, 2H, $\mathrm{CH}_{2}$ ); IR (KBr) 2930, 2876, 1529, 1323, 1307, 1126, $1096 \mathrm{~cm}^{-1}$; MS (EI, $\left.70 \mathrm{eV}\right) \mathrm{m} / \mathrm{z}$ (rel intensity) $115\left(\mathrm{M}^{+}, 100\right), 100\left(\left[\mathrm{M}-\mathrm{CH}_{3}\right]^{+}, 26\right), 82\left([\mathrm{M}-\mathrm{SH}]^{+}, 48\right)$.

\section{Azepane-2-thione (10c) ${ }^{3}$}

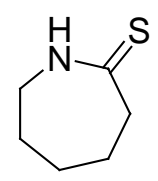

The reaction was carried out for $2 \mathrm{~h}$ according to the general thermal thionation procedure.

${ }^{1} \mathrm{H}-\mathrm{NMR}:\left(\mathrm{CDCl}_{3}, 200 \mathrm{MHz}\right) \delta 9.05$ (br s, 1H, N-H), 3.40-3.32 (m, 2H, $\left.\mathrm{CH}_{2}\right), 3.00-2.94\left(\mathrm{~m}, 2 \mathrm{H}, \mathrm{CH}_{2}\right)$, 1.85-1.56 (m, 6H); IR (KBr) 3182, 2936, 2927, 1560, 1440, 1409, 1369, 1348, 1333, 1315, 1261, 1175, 1114, 1070, 980, $779 \mathrm{~cm}^{-1}$; MS (EI, $\left.70 \mathrm{eV}\right) \mathrm{m} / z$ (rel intensity) $129\left(\mathrm{M}^{+}, 100\right), 114\left([\mathrm{M}-\mathrm{NH}]^{+}, 45\right)$, $101\left([\mathrm{M}-28]^{+}, 49\right), 96\left([\mathrm{M}-\mathrm{SH}]^{+}, 65\right), 41(84), 39(68), 58(26), 71$ (38), 68 (35). 
$N_{N H_{2}}^{S}$

The reaction was carried out for $1.5 \mathrm{~h}$ according to the general thermal thionation procedure.

${ }^{1} \mathrm{H}-\mathrm{NMR}:\left(\mathrm{CDCl}_{3}, 200 \mathrm{MHz}\right) \delta 7.86(\mathrm{~m}, 2 \mathrm{H}, \mathrm{Ar}-\mathrm{H}), 7.55-7.35$ (m, 3H, Ar-H); IR (KBr) 3363, 3285, 3161, 1623, 1450, 1400, 1325, 1280, 887, 774, 708, 688, 637, $568 \mathrm{~cm}^{-1}$; MS (EI, $\left.70 \mathrm{eV}\right) \mathrm{m} / \mathrm{z}$ (rel intensity) $137\left(\mathrm{M}^{+}, 100\right), 121\left(\left[\mathrm{M}-\mathrm{NH}_{2}\right]^{+}, 35\right), 104\left([\mathrm{M}-\mathrm{SH}]^{+}, 54\right)$.

\section{Pyridine-3-carbothioamide (10e) $)^{3}$}<smiles>NC(=S)c1cccnc1</smiles>

The reaction was carried out for $2 \mathrm{~h}$ according to the general thermal thionation procedure.

${ }^{1} \mathrm{H}-\mathrm{NMR}:\left(\mathrm{CD}_{3} \mathrm{COCD}_{3}, 200 \mathrm{MHz}\right) \delta 9.20\left(\mathrm{br} \mathrm{s}, 2 \mathrm{H}, \mathrm{NH}_{2}\right), 9.12(\mathrm{~d}, J=1.4 \mathrm{~Hz}, 1 \mathrm{H}, \mathrm{Ar}-\mathrm{H}), 8.67(\mathrm{dd}, J=$ 4.8, 1.4 Hz, 1H, Ar-H) $8.30(\mathrm{dt}, J=8.0,1.4 \mathrm{~Hz}, 1 \mathrm{H}, \mathrm{Ar}-\mathrm{H}), 7.4$ (dd, $J=8.0,4.8 \mathrm{~Hz}, 1 \mathrm{H}, \mathrm{Ar}-\mathrm{H})$; IR (KBr) 3236, 3029, 2961, 2810, 1677, 1588, 1457, 1400, 1311, 1029, 913, 736, 700, $630 \mathrm{~cm}^{-1}$; MS (EI, $70 \mathrm{eV}) \mathrm{m} / z$ (rel intensity) $138\left(\mathrm{M}^{+}, 100\right), 122\left(\left[\mathrm{M}-\mathrm{NH}_{2}\right]^{+}, 12\right), 105\left([\mathrm{M}-\mathrm{SH}]^{+}, 65\right), 78\left(\mathrm{Pyr}^{+}, 20\right)$.

\section{6-methyl-2H-1,4-benzoxazine-3(4H)-thione (10f)}<smiles>Cc1ccc2c(c1)NC(=S)CO2</smiles>

The reaction was carried out for $1 \mathrm{~h}$ according to the general thermal thionation procedure.

${ }^{1} \mathrm{H}-\mathrm{NMR}:\left(\mathrm{CDCl}_{3}, 200 \mathrm{MHz}\right) \delta 10.45$ (br s, 1H, N-H), 6.86 (m, 2H, Ar-H), 6.74 (m, 1H, Ar-H), 4.85 (s, 2H, $\mathrm{CH}_{2}$ ), 2.29 (s, 3H, $\left.\mathrm{CH}_{3}\right) ;{ }^{13} \mathrm{C}-\mathrm{NMR}:\left(\mathrm{CDCl}_{3}, 50 \mathrm{MHz}\right) \delta 191.7$ (CS), 143.3 (C), 132.7 (C), 126.7 $(\mathrm{CH})$, 125,6 (C), $116.8(\mathrm{CH}), 116.3(\mathrm{CH}), 72.9\left(\mathrm{CH}_{2}\right), 20.8\left(\mathrm{CH}_{3}\right), \mathrm{R}(\mathrm{KBr})$ 3185, 3133, 3031, 2918, 1557, 1509, 1392, 1274, 1231, 1138, 1106, 1009, 860, 808, 788, $644 \mathrm{~cm}^{-1}$; MS (EI, $\left.70 \mathrm{eV}\right) \mathrm{m} / z$ (rel intensity) $179\left(\mathrm{M}^{+}, 100\right), 146\left([\mathrm{M}-\mathrm{SH}]^{+}, 58\right), 134\left([\mathrm{M}-\mathrm{CSH}]^{+}, 10\right) . \mathrm{HRMS}(\mathrm{EI}, 70 \mathrm{eV})$ calcd. for 179.0405, found: 179.0409 . 


\section{Thioacetanilide $(\mathbf{1 0 g})^{3}$}<smiles>CC(=S)Nc1ccccc1</smiles>

The reaction was carried out for $4 \mathrm{~h}$ according to the general thermal thionation procedure.

Mixture of Z/E isomers. ${ }^{1} \mathrm{H}-\mathrm{NMR}:\left(\mathrm{CDCl}_{3}, 200 \mathrm{MHz}\right) \delta 9.95$ (br s, 0.7H, N-H), 9.19 (br s, 0.9H, N-H), 7.68-7.62 (m, 1.7 H, Ar-H), 7.45-7.32 (m, 3.9H, Ar-H), 7.29-7.12 (m, 2.6H, Ar-H), 2.70 (s, 3H, N$\mathrm{CH}_{3}$ ); 2.49 (s, 2.3H, CH $)$; IR (KBr) 3167, 3118, 3001, 2967, 1593, 1537, 1494, 1448, 1368, 1151,706 $\mathrm{cm}^{-1}$; MS (EI, $\left.70 \mathrm{eV}\right) \mathrm{m} / z$ (rel intensity) $151\left(\mathrm{M}^{+}, 100\right), 150\left([\mathrm{M}-\mathrm{H}]^{+}, 71\right), 136\left(\left[\mathrm{M}-\mathrm{CH}_{3}\right]^{+}, 4\right), 118([\mathrm{M}-$ $\left.\mathrm{SH}]^{+}, 31\right)$.

\section{General procedures for use fluorous Lawesson's reagent $(8)$ in microwave.}

The preparation of thiocoumarin $(\mathbf{1 0 h})$ is a representative of the method employed.

A mixture of coumarin $(\mathbf{9 h}, 29.22 \mathrm{mg}, 0.2 \mathrm{mmol})$ and fluorous Lawesson's reagent 8 (138 $\mathrm{mg}, 0.1$ mmol) was taken in a glass tube and mixed thoroughly with a spatula. The glass tube was then placed in the microwave oven and irradiated for 3 minutes. On completion of the reaction, followed by TLC, the colored material was mixed with $2.0 \mathrm{~g}$ of neutral aluminium oxide (Brockmann II, neutral) and was placed on a short column packed with FluoroFlash ${ }^{\mathrm{TM}}$ SPE silica $(2.0 \mathrm{~g})$. The column was eluted with methanol/water $8 / 2$ and evaporated to dryness to give the pure $\mathbf{1 0 h}$.

\section{Thioacetanilide $(\mathbf{1 0 g})^{3}$}<smiles>CC(=S)Nc1ccccc1</smiles>

The reaction was carried out for 3 minutes according to the general method. 


\section{Thiocoumarin $(\mathbf{1 0 h})^{6}$}<smiles>S=c1ccc2ccccc2o1</smiles>

The reaction was carried out for 3 minutes according to the general method .

${ }^{1} \mathrm{H}-\mathrm{NMR}:\left(\mathrm{CDCl}_{3}, 200 \mathrm{MHz}\right) \delta$ 7.65-7.45 (m, 3H, Ar-H), $7.45(\mathrm{~d}, J=9.2 \mathrm{~Hz}, 1 \mathrm{H},=\mathrm{CH}), 7.37-7.27$ (m, 1H, Ar-H), 7.22 (d, $J=9.2 \mathrm{~Hz}, 1 \mathrm{H},=\mathrm{CH})$; IR (KBr) 1607, 1552, 1415, 1252, 1206, 1194, 1127, 1094, 816, 764, 749, 592, $456 \mathrm{~cm}^{-1}$; MS (EI, $\left.70 \mathrm{eV}\right) \mathrm{m} / z$ (rel intensity) $162\left(\mathrm{M}^{+}, 90\right), 118$ ([M-CS] $\left.]^{+}, 100\right), 90$ (18), 89 (18).

Thiobenzophenone (10i) ${ }^{7}$

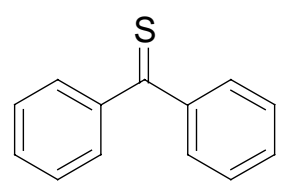

The reaction was carried out for 3 minutes according to the general procedure. The column was eluted with anhydrous acetonitrile to give the product. The product purity was $80 \%$ based upon NMR mesaurement (20\% starting material left).

${ }^{1} \mathrm{H}-\mathrm{NMR}:\left(\mathrm{CDCl}_{3}, 200 \mathrm{MHz}\right) \delta$ 7.75-7.69 (m, 4H, Ar-H), 7.61-7.52 (m, 2H, Ar-H), 7.44-7.34 (m, 4H, Ar-H);

${ }^{13} \mathrm{C}-\mathrm{NMR}:\left(\mathrm{CDCl}_{3}, 50 \mathrm{MHz}\right) \delta 238.5(\mathrm{CS}), 147.4,132.1,129.7,128.1$;

MS (EI, 70 eV) m/z (rel intensity): 198, $\left(\mathrm{M}^{+}, 95\right), 165$ ([M-SH] $\left.]^{+}, 100\right), 121\left([\mathrm{M}-\mathrm{Ph}]^{+}, 90\right), 77\left(\mathrm{Ph}^{+}, 38\right)$ 
To a mixture of the corresponding $\beta$-ketoamide $\mathbf{9 j , k}(0.1 \mathrm{mmol})$, phenylhydrazine hydrochloride (16 $\mathrm{mg}, 0.11 \mathrm{mmol})$ and fluorous Lawesson's reagent $8(152 \mathrm{mg}, 0.11 \mathrm{mmol})$ was added $1.0 \mathrm{~mL}$ of dry THF/pyridine (95/5) solution via syringe. The reaction was stirred at room temperature for $15 \mathrm{~min}$ and the flask was transferred to a heating block. After the vial was kept at $55^{\circ} \mathrm{C}$ for $14 \mathrm{~h}, 2.0 \mathrm{~g}$ of neutral aluminium oxide (Brockmann II, neutral) was added and the solvent was evaporated. The resulting solid was placed on a short column packed with FluoroFlash ${ }^{\mathrm{TM}}$ SPE silica $(2.0 \mathrm{~g})$. The column was eluted with methanol/water $8 / 2$ product evaporated to dryness to give the pure product $\mathbf{1 0 j} \mathbf{j} \mathbf{k}$.

\section{N,N-diethyl-3-methyl-1-phenyl-1H-pyrazol-5-amine (10j) $)^{8,9}$}

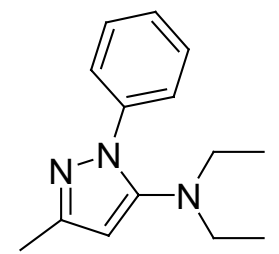

${ }^{1} \mathrm{H}-\mathrm{NMR}:\left(\mathrm{CDCl}_{3}, 200 \mathrm{MHz}\right) \delta 7.68(\mathrm{~m}, 2 \mathrm{H}, \mathrm{Ar}-\mathrm{H}), 7.41(\mathrm{~m}, 2 \mathrm{H}, \mathrm{Ar}-\mathrm{H}), 7.28$ (m, 1H, Ar-H), 5.72 (s, 1H, Pyraz-H), 2.90 (q, $J=7.0 \mathrm{~Hz}, 4 \mathrm{H}, \mathrm{N}-\mathrm{CH}_{2}$ ), 2.32 (s, 3H, Pyraz- $\mathrm{CH}_{3}$ ), 0.99 (t, J=7.0 Hz, 6H, $\mathrm{CH}_{2^{-}}$ $\mathrm{CH}_{3}$ ); IR (KBr) 2973, 2933, 1599, 1554, 1504, 1454, 1382, 1364, 1206, 763, $693 \mathrm{~cm}^{-1}$; MS (EI, 70 eV) $m / z$ (rel intensity) $229\left(\mathrm{M}^{+}, 85\right), 214\left(\left[\mathrm{M}-\mathrm{CH}_{3}\right]^{+}, 100\right), 200\left(\left[\mathrm{M}-\mathrm{C}_{2} \mathrm{H}_{5}\right]^{+}, 10\right), 186(8), 184$ (9), 77 (24).

\section{3-methyl-N,1-diphenyl-1H-pyrazol-5-amine (10k) $)^{8,10}$}

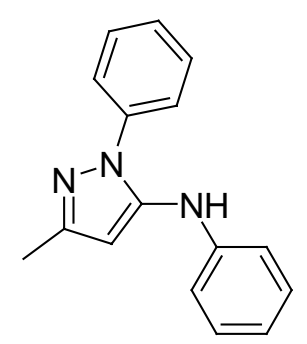

${ }^{1} \mathrm{H}-\mathrm{NMR}:\left(\mathrm{CDCl}_{3}, 200 \mathrm{MHz}\right)$ \% 7.58-7.21 (m, 7H, Ar-H), 6.99-6.92 (m, 3H, Ar-H), 5.99 (s, 1H, PyrazH), 2.33 (s, 3H, Pyraz-CH ${ }_{3}$; IR (KBr) 3229, 1594, 1561, 1498, 1417, 1363, 1304, 1260, 750, $696 \mathrm{~cm}^{-}$ '; MS (EI, $70 \mathrm{eV}) \mathrm{m} / z$ (rel intensity) $249\left(\mathrm{M}^{+}, 100\right), 116$ (9), 91 (6), 77 (30). 


\section{Spectra:}

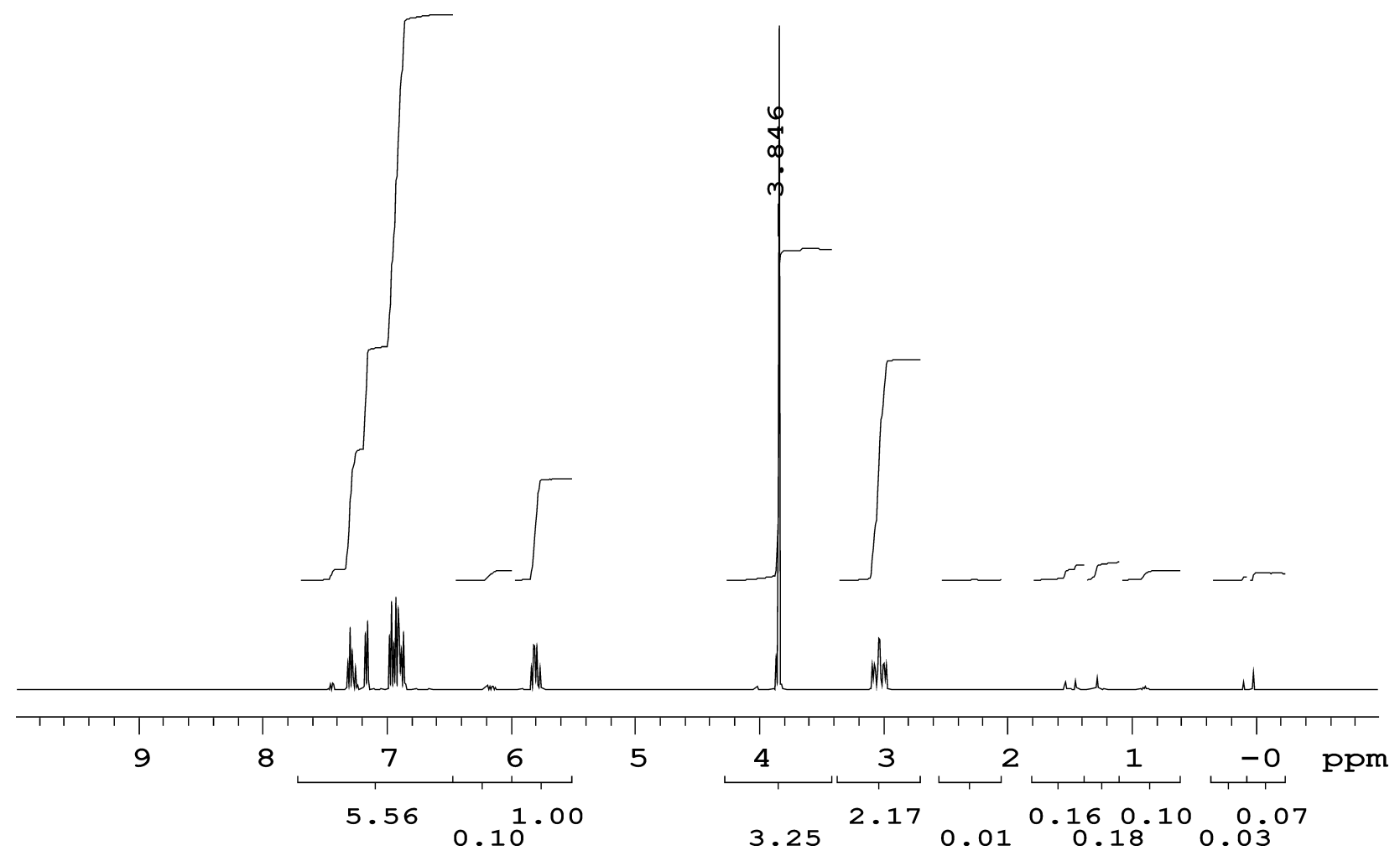

Figure S1. ${ }^{1} \mathrm{H}$ NMR spectra of compound $\mathbf{I}$. 


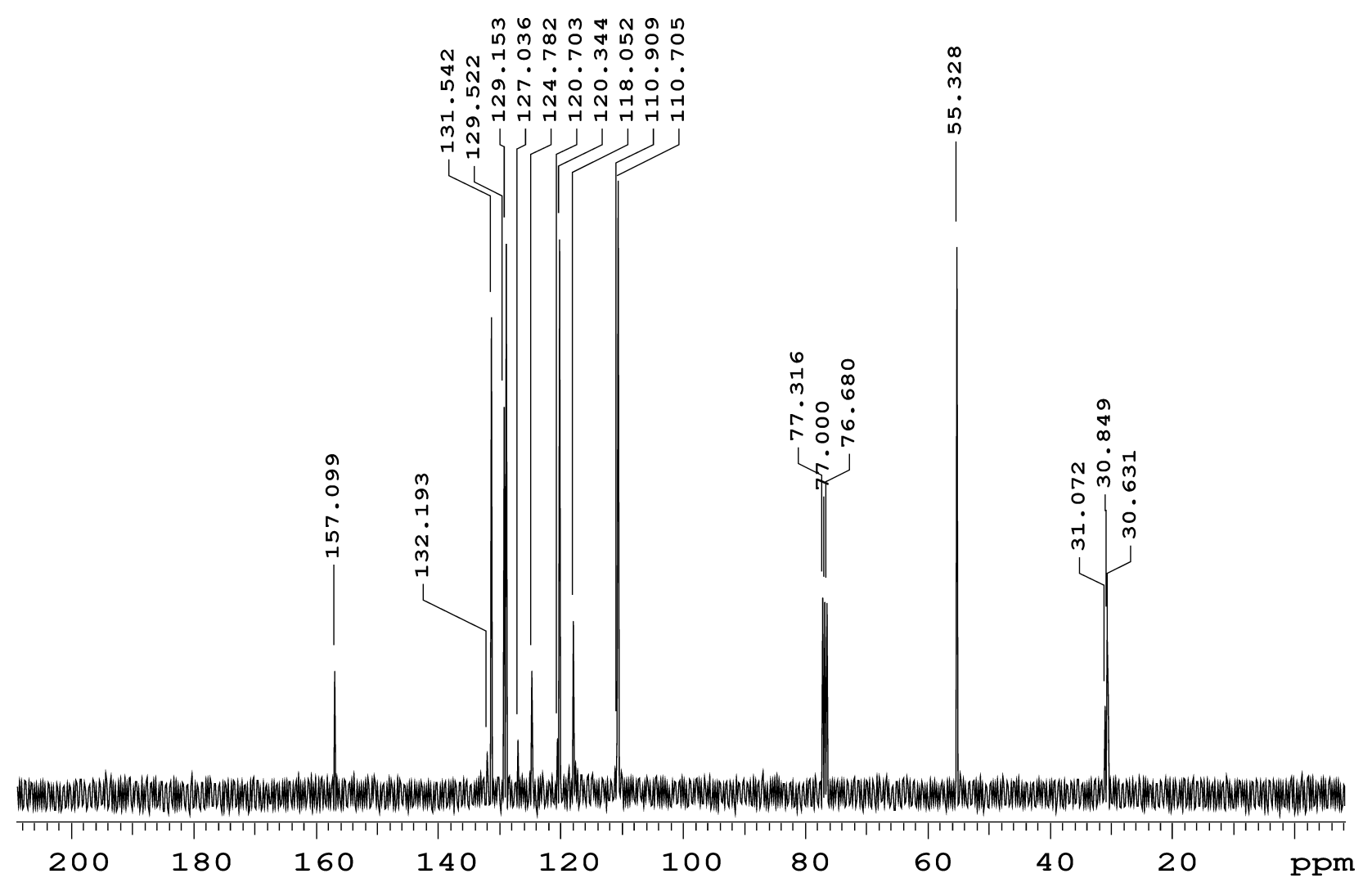

Figure S2. ${ }^{13} \mathrm{C}$ NMR spectra of compound I

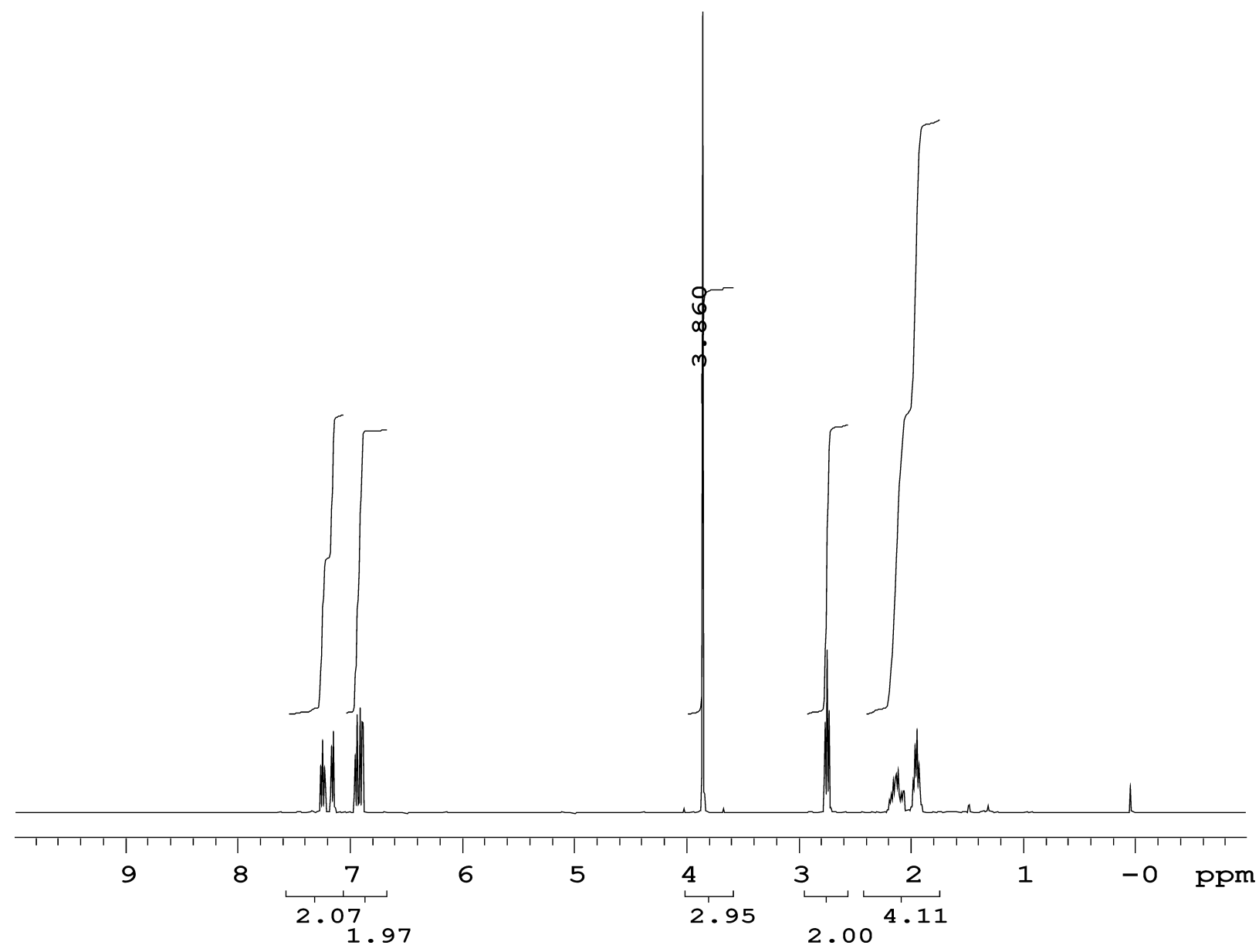


Figure S3. ${ }^{1} \mathrm{H}$ NMR spectra of compound 3a.

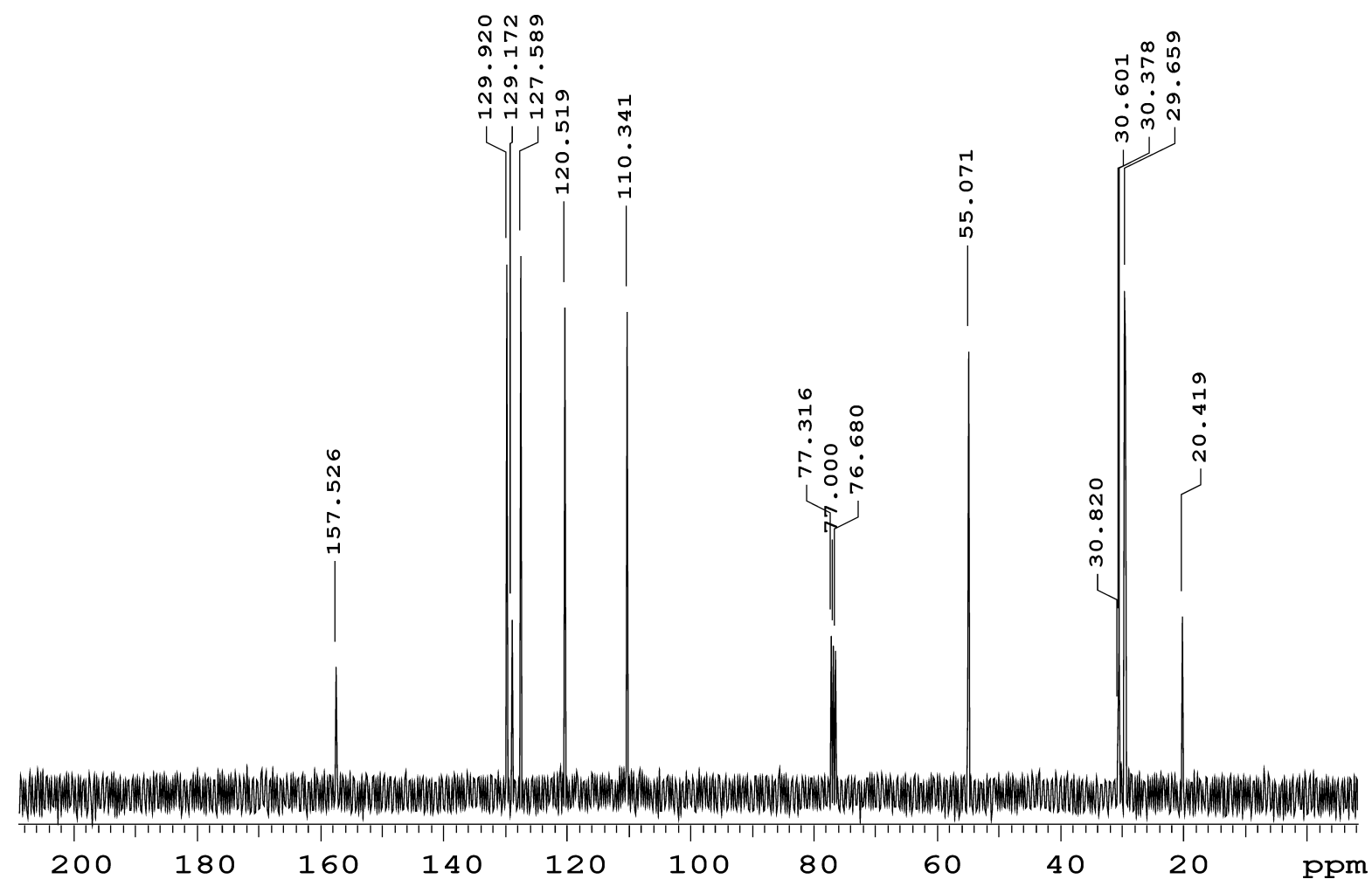

Figure S4. ${ }^{13} \mathrm{C}$ NMR spectra of compound $\mathbf{3 a}$ 


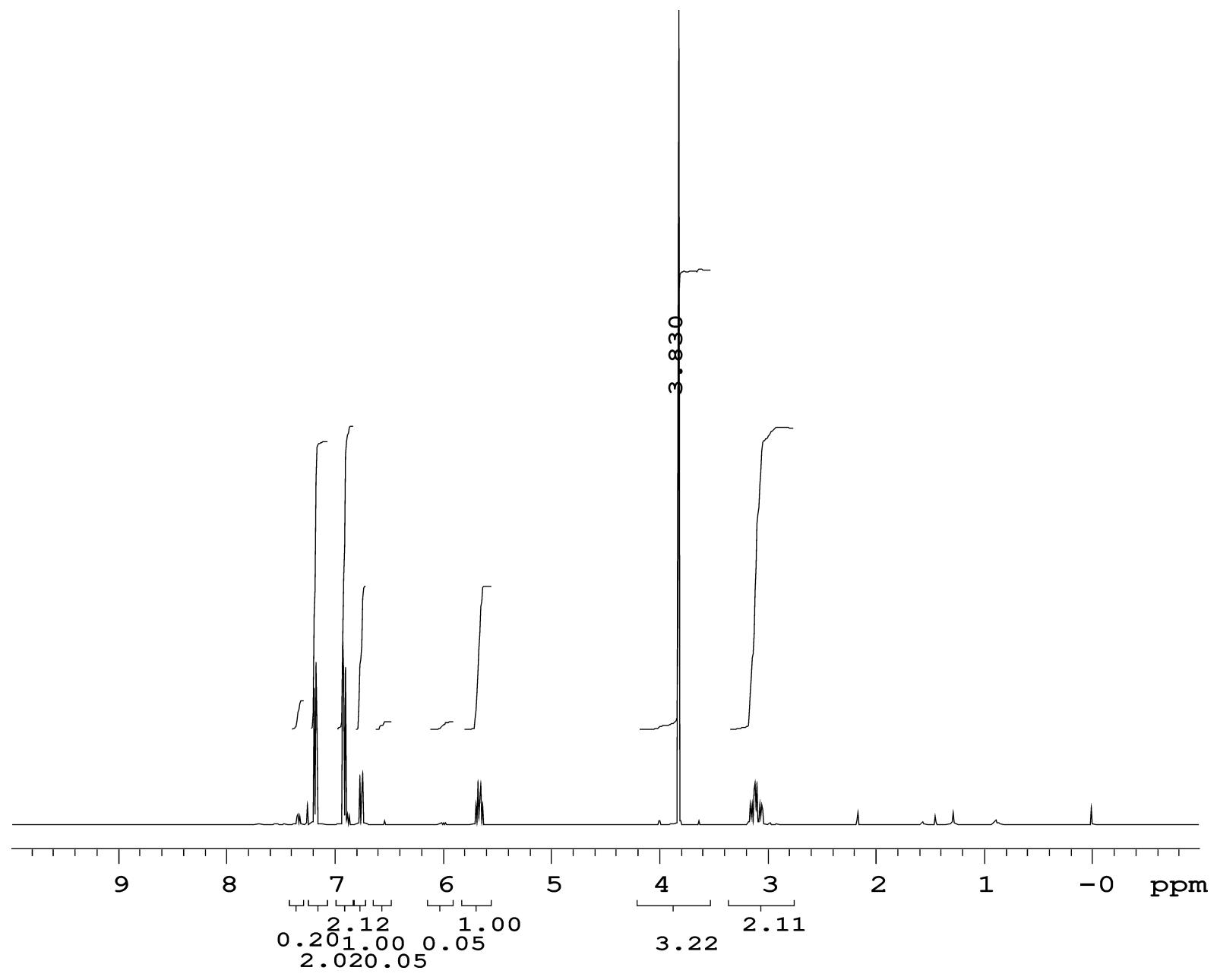

Figure S5. ${ }^{1} \mathrm{H}$ NMR spectra of compound II.

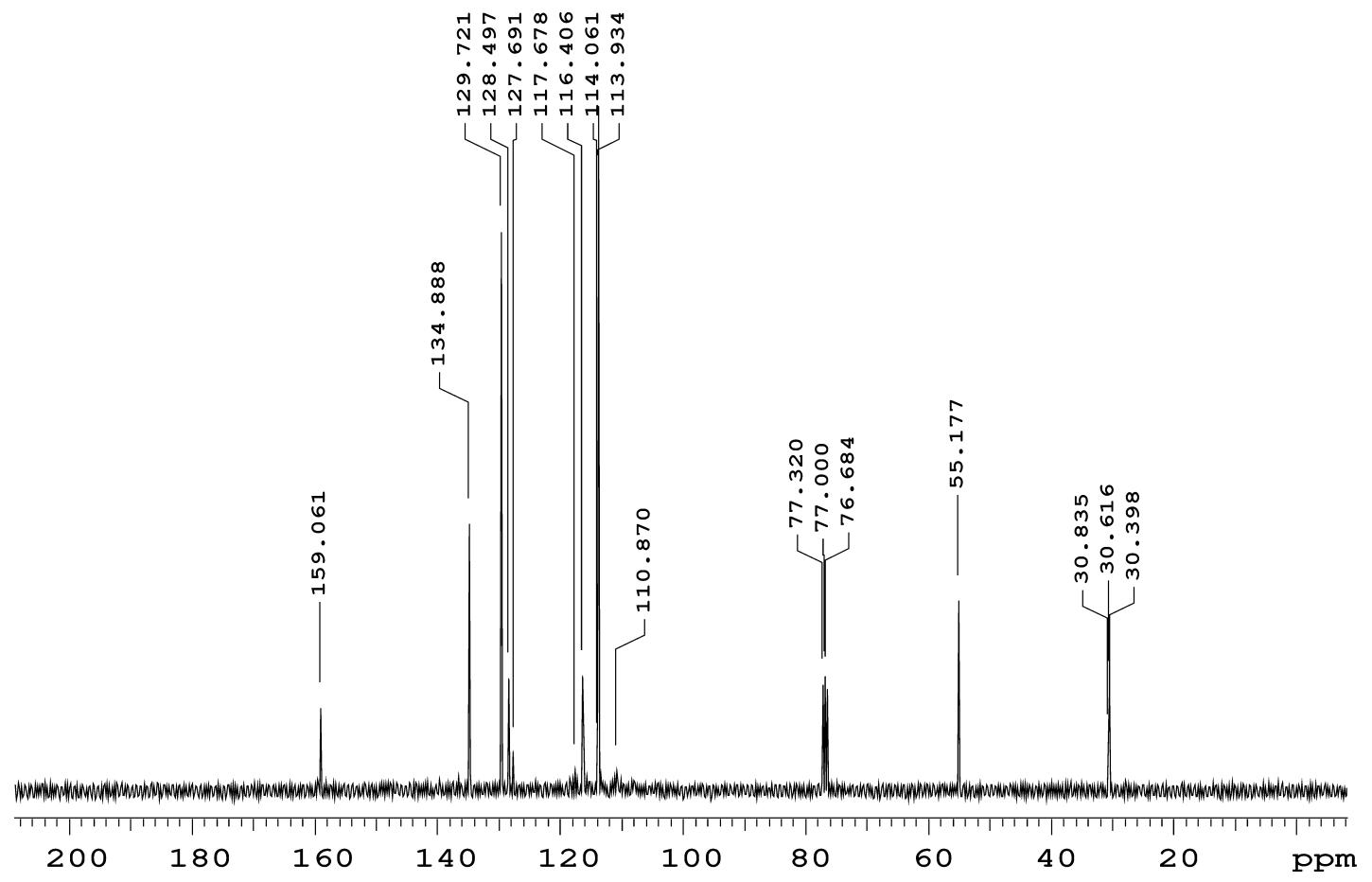

Figure S6. ${ }^{13} \mathrm{C}$ NMR spectra of compound II 


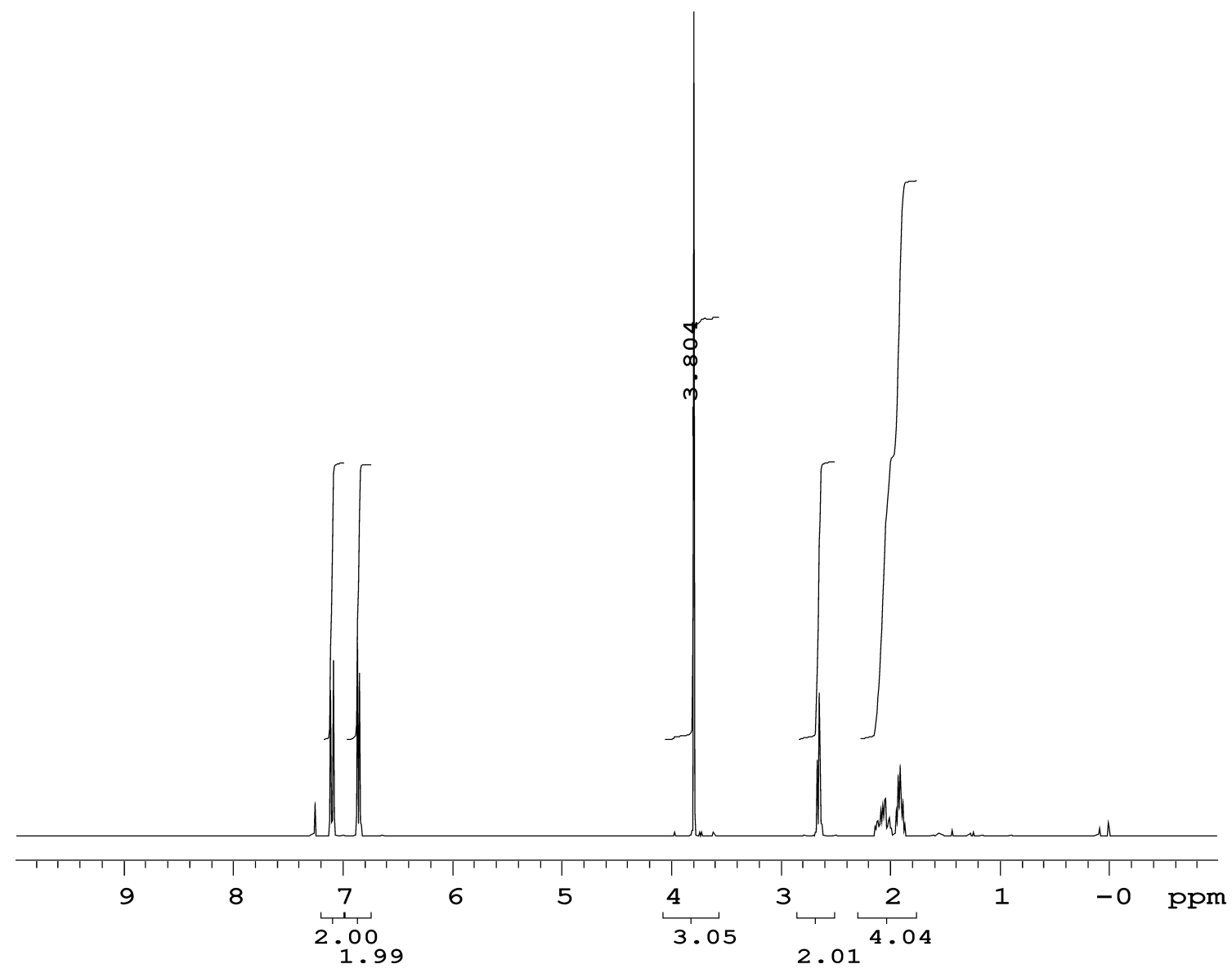

Figure S7. ${ }^{1} \mathrm{H}$ NMR spectra of compound $\mathbf{3 b}$.

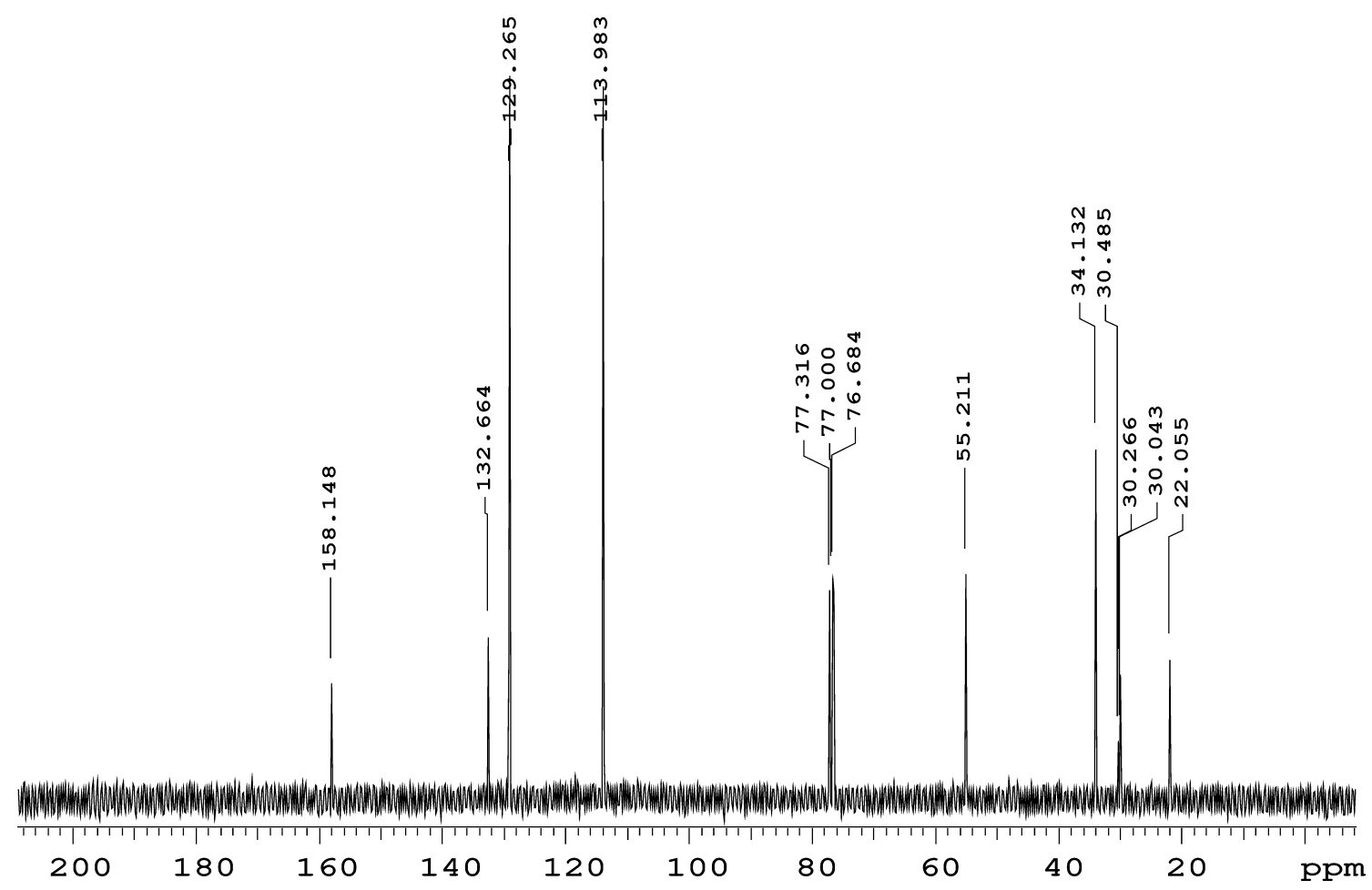

Figure S8. ${ }^{13} \mathrm{C}$ NMR spectra of compound $\mathbf{3 b}$ 


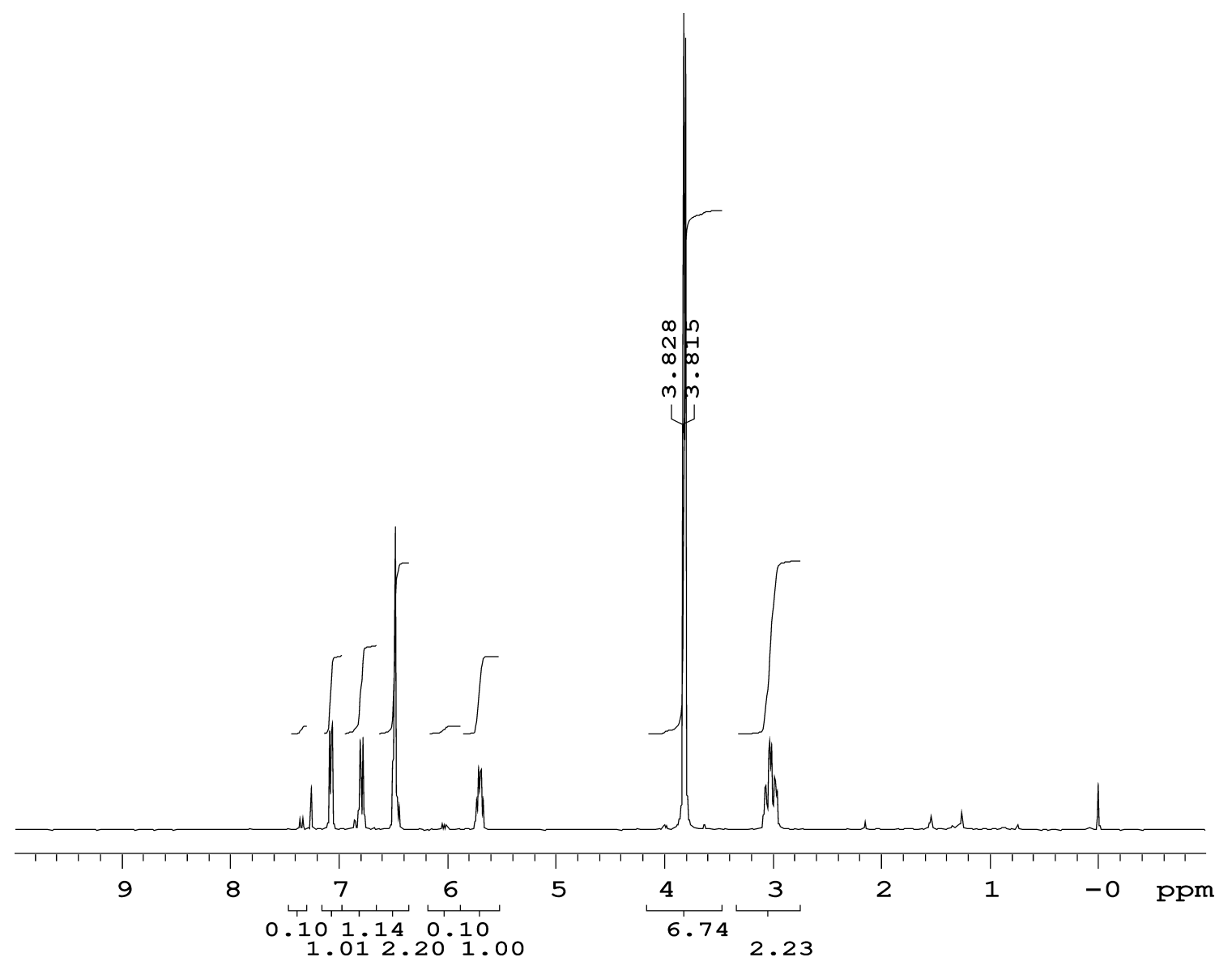

Figure S9. ${ }^{1} \mathrm{H}$ NMR spectra of compound III.

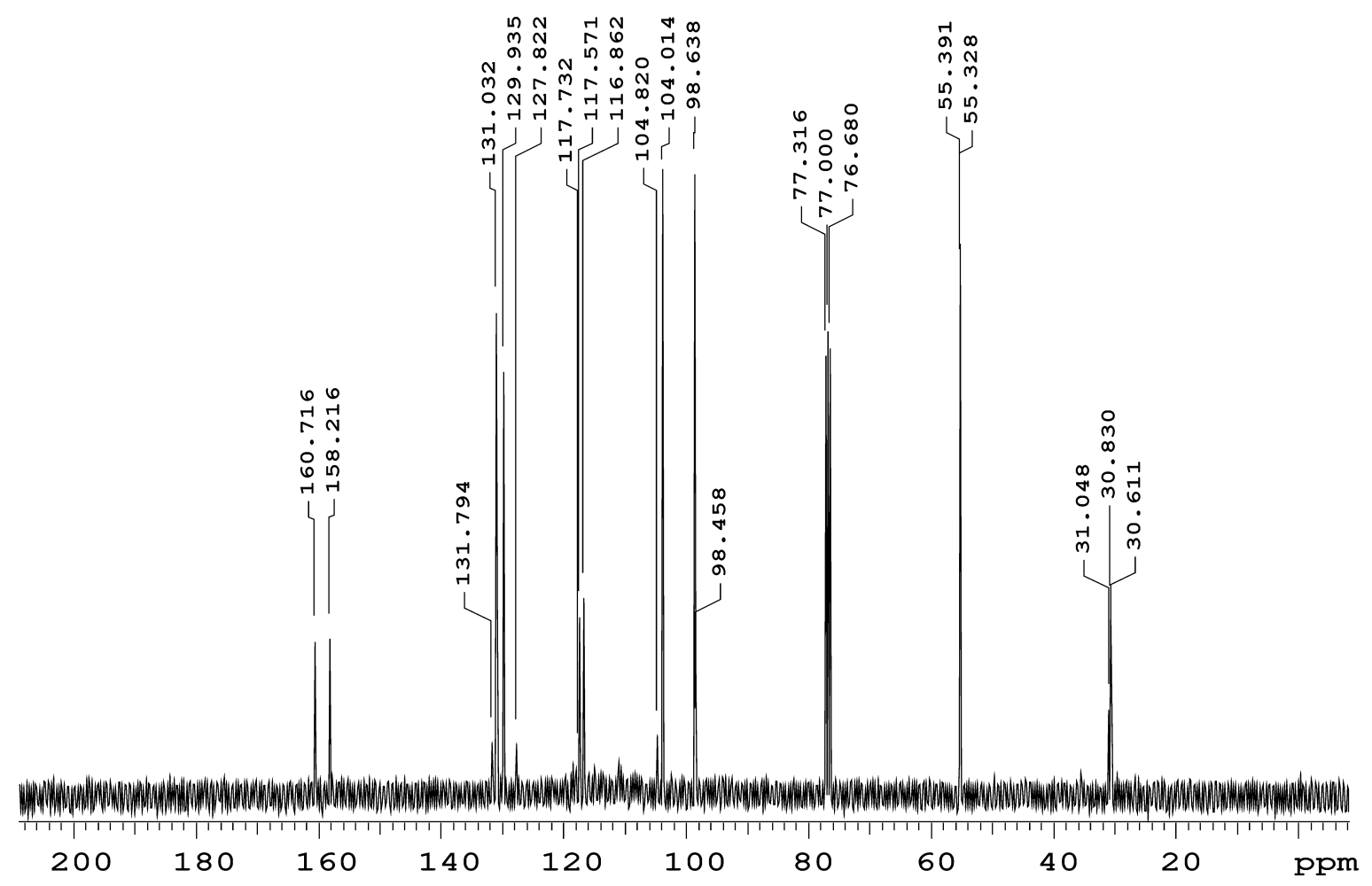

Figure S10. ${ }^{13} \mathrm{C}$ NMR spectra of compound III 


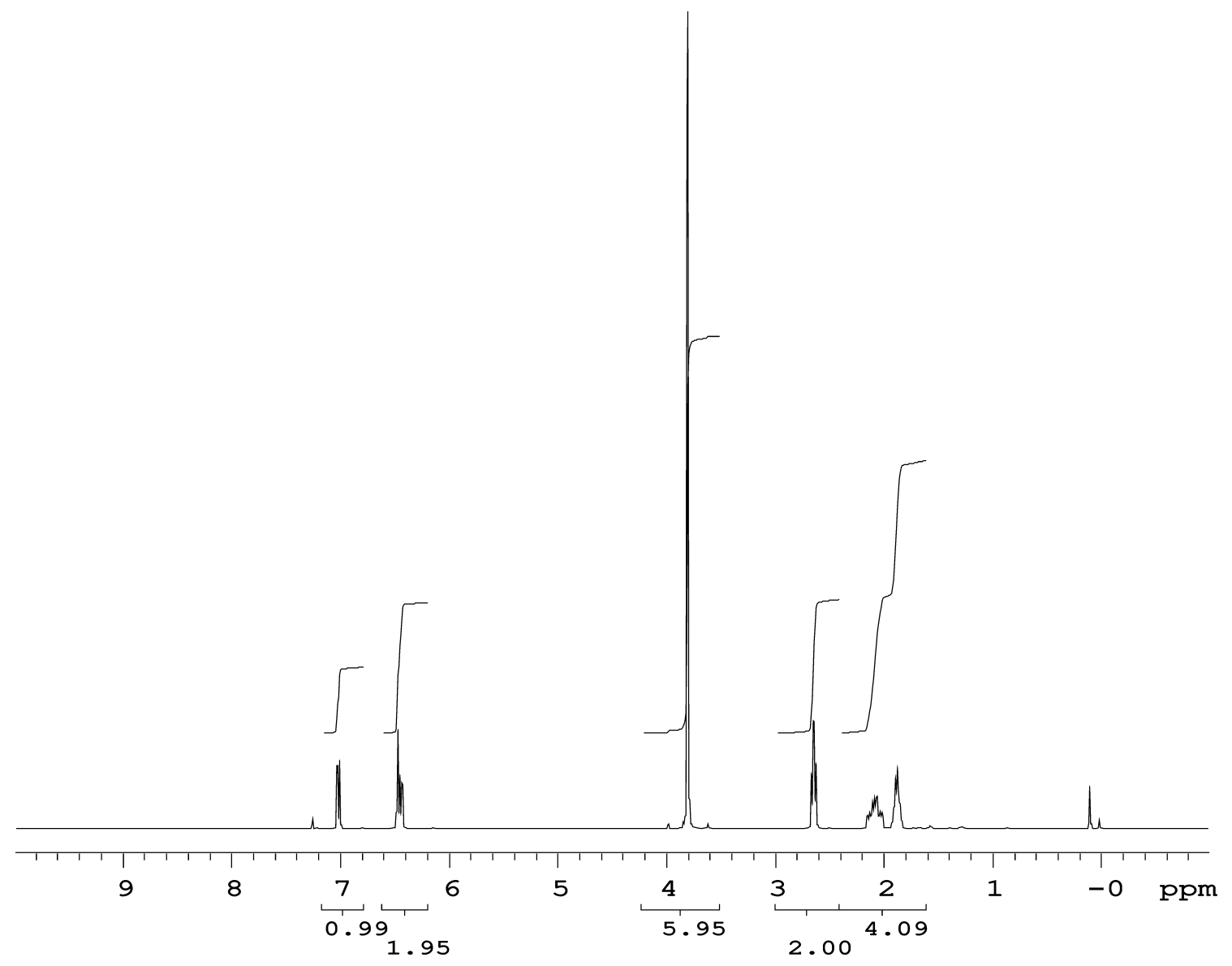

Figure S11. ${ }^{1} \mathrm{H}$ NMR spectra of compound 3c

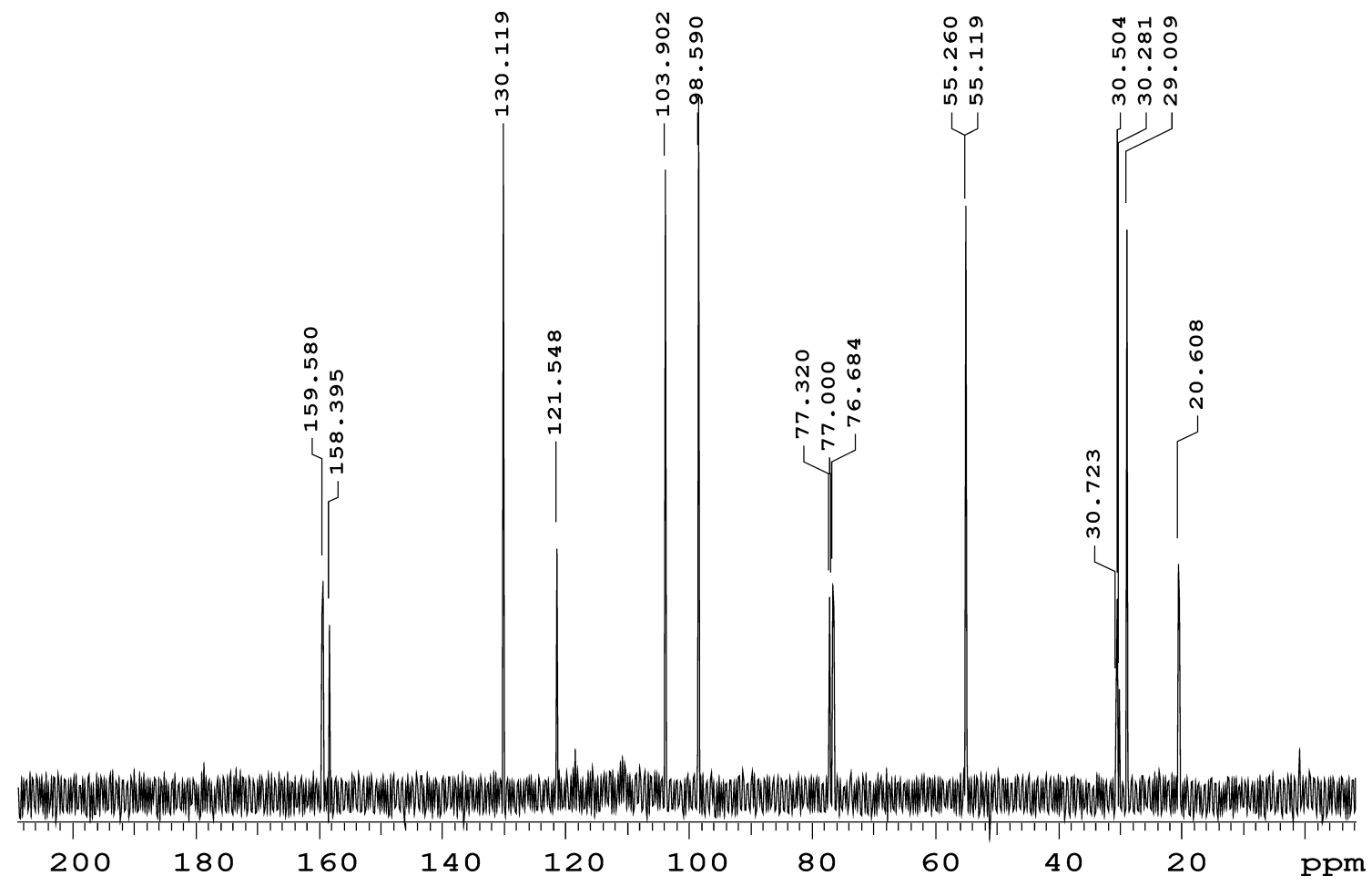

Figure S12. ${ }^{13} \mathrm{C}$ NMR spectra of compound 3c 


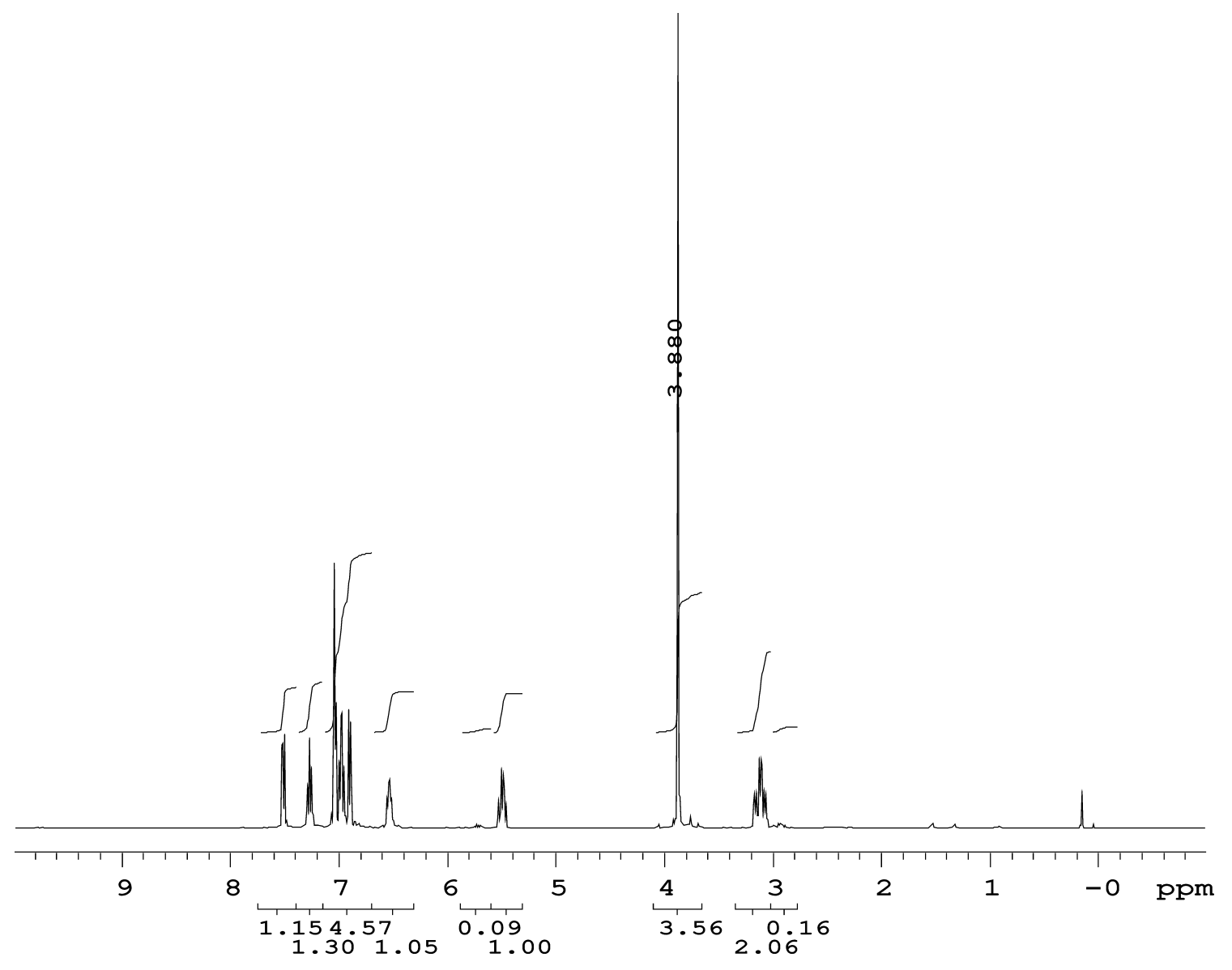

Figure S13. ${ }^{1} \mathrm{H}$ NMR spectra of compound 6

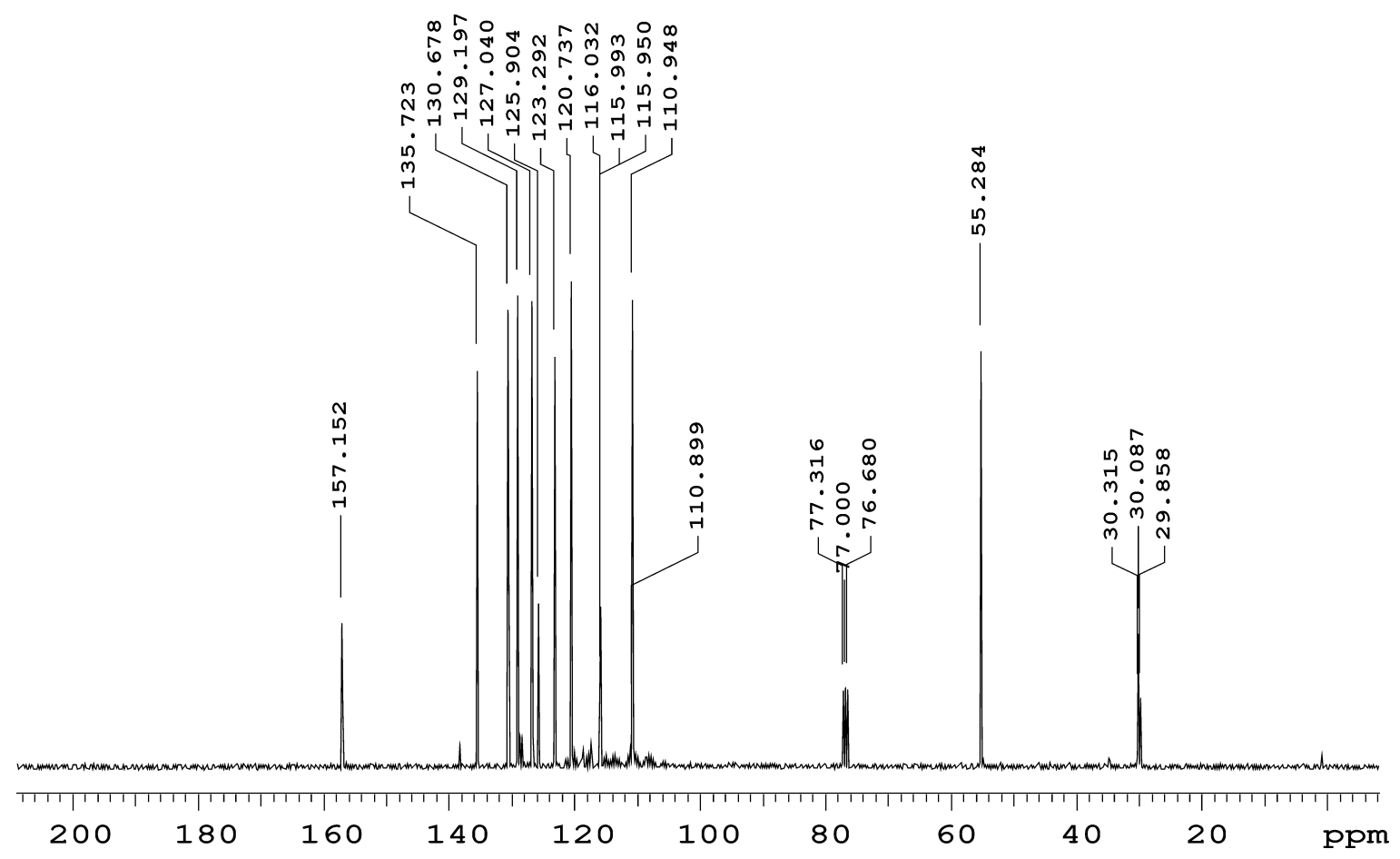

Figure S14. ${ }^{13} \mathrm{C}$ NMR spectra of compound 6 


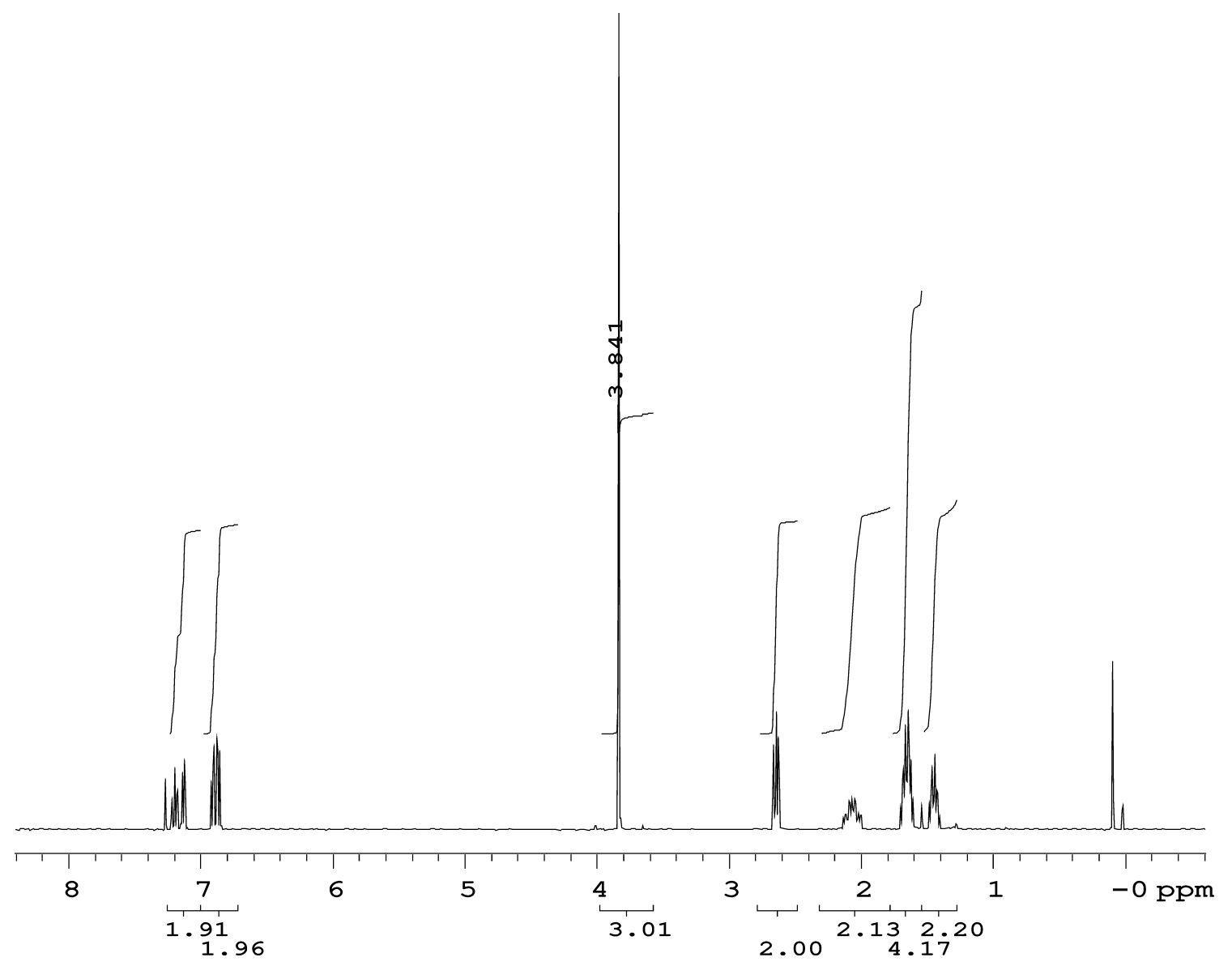

Figure S15. ${ }^{1} \mathrm{H}$ NMR spectra of compound 7

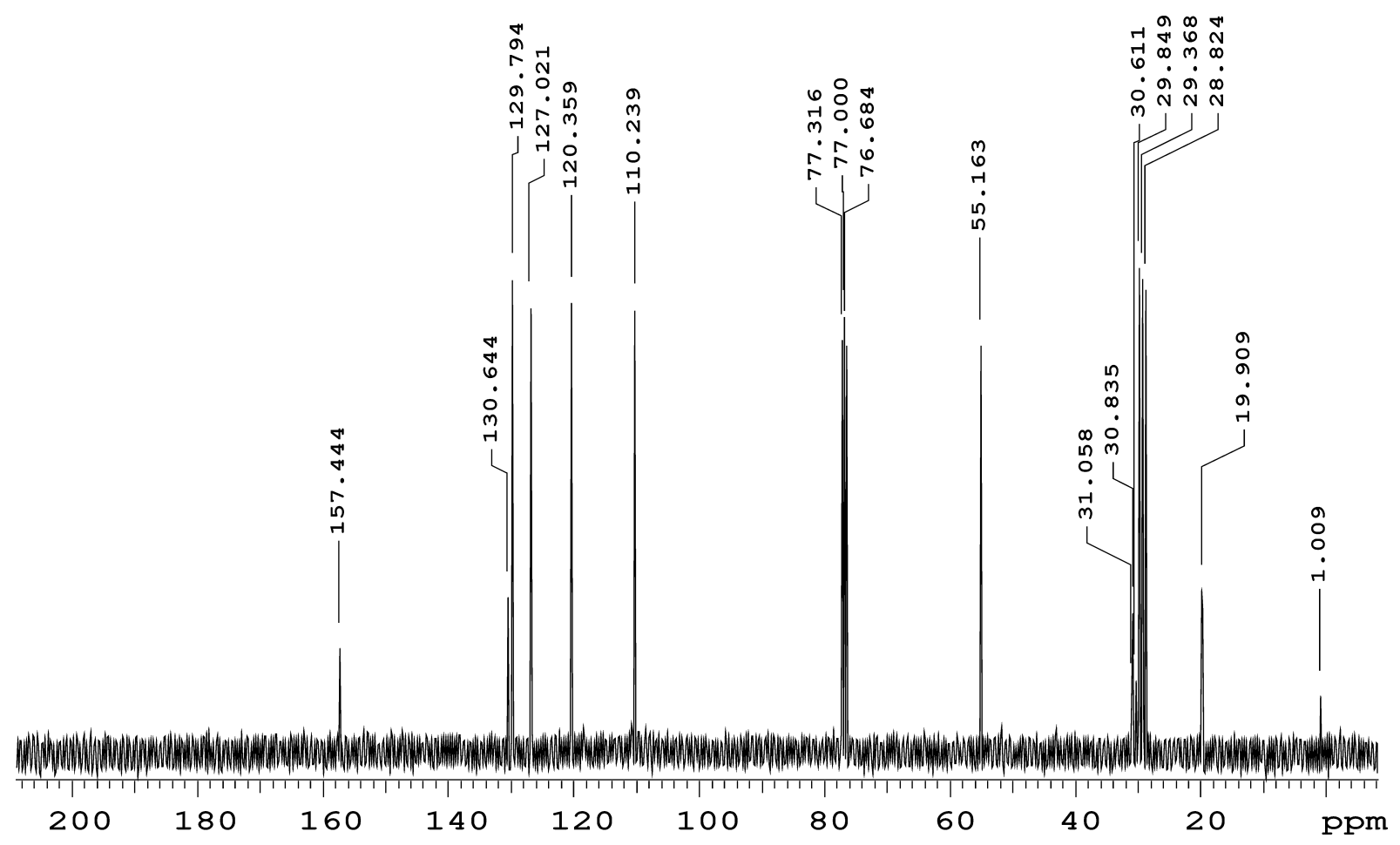

Figure S16. ${ }^{13} \mathrm{C}$ NMR spectra of compound 7 


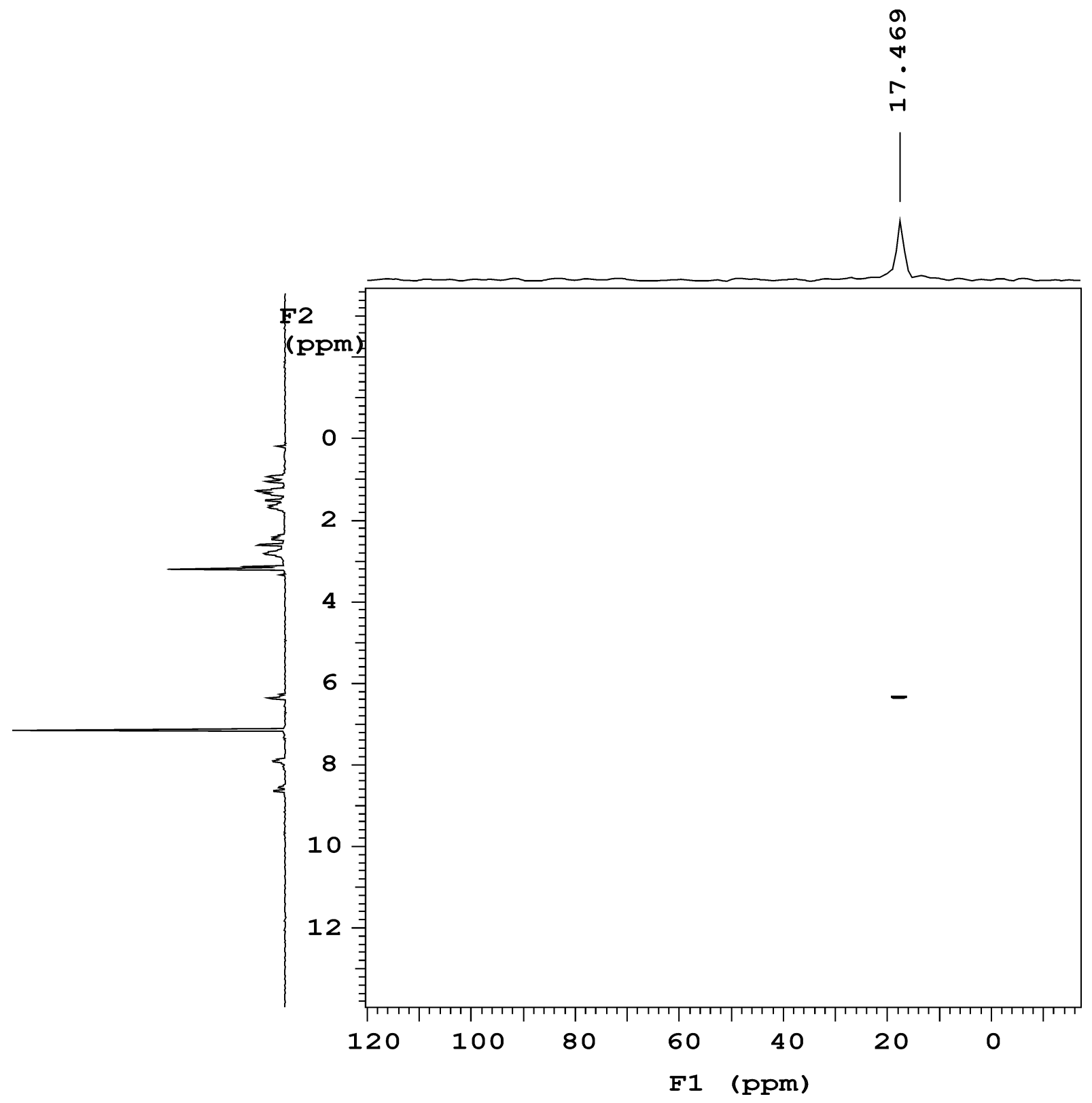

Figure S17. ${ }^{1} \mathrm{H}_{-}{ }^{31} \mathrm{P}-\mathrm{gHMQC}$ spectrum $\left(\mathrm{C}_{6} \mathrm{D}_{6}, 25{ }^{\circ} \mathrm{C}, 400 \mathrm{MHz}\right)$ of compound 8. The three bond $\mathrm{P}-\mathrm{H}$ correlation (15 Hz optimized) is shown as a crosspeak. The horizontal and vertical scales represent the ${ }^{31} \mathrm{P}$ and ${ }^{1} \mathrm{H}$ chemical shift dimensions respectively. 


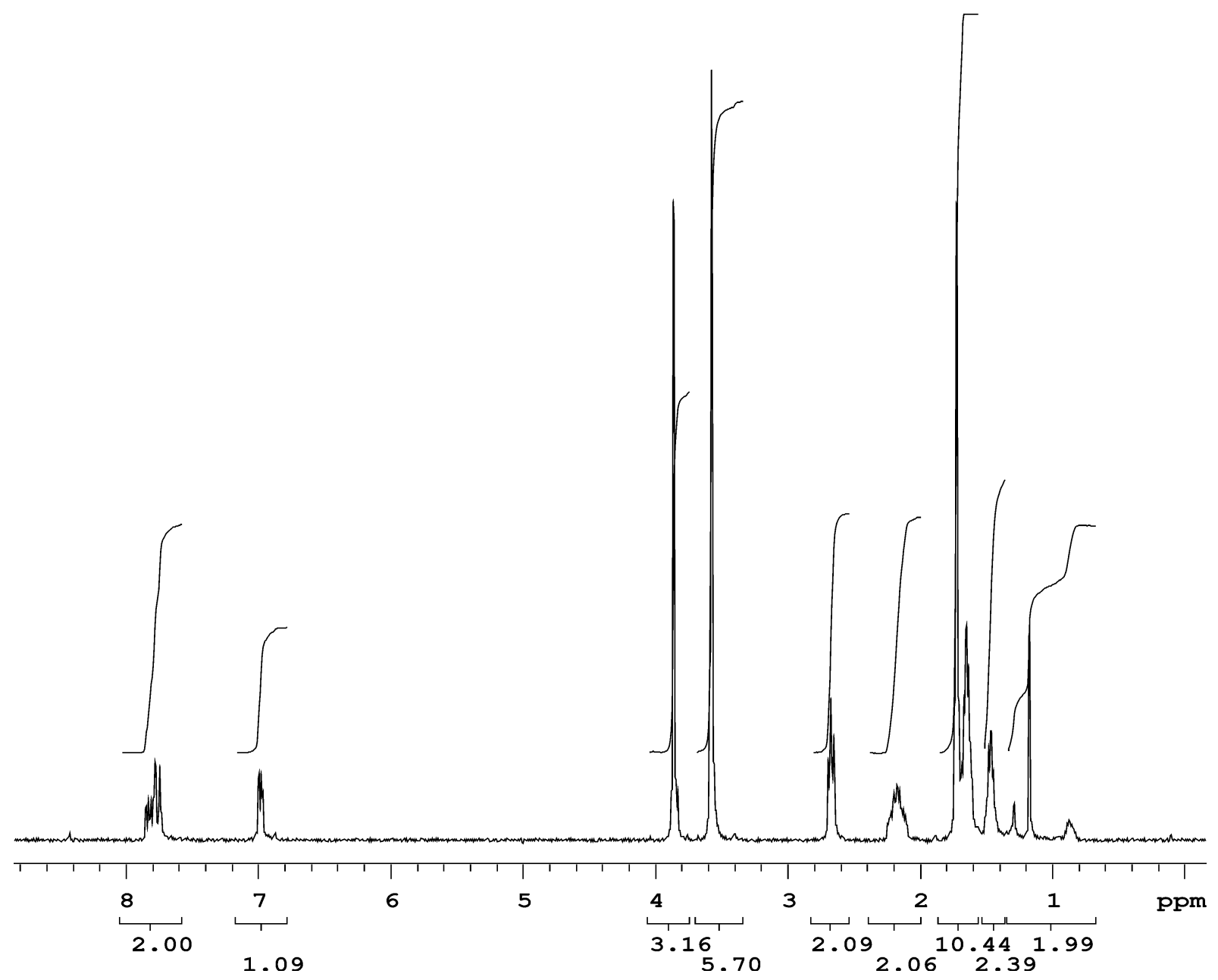

Figure S18. ${ }^{1} \mathrm{H}-\mathrm{NMR}$ spectrum $\left(25^{\circ} \mathrm{C}, 400 \mathrm{MHz}\right)$ of compound 8 when dissolved in $\mathrm{THF}-\mathrm{d}_{8}$
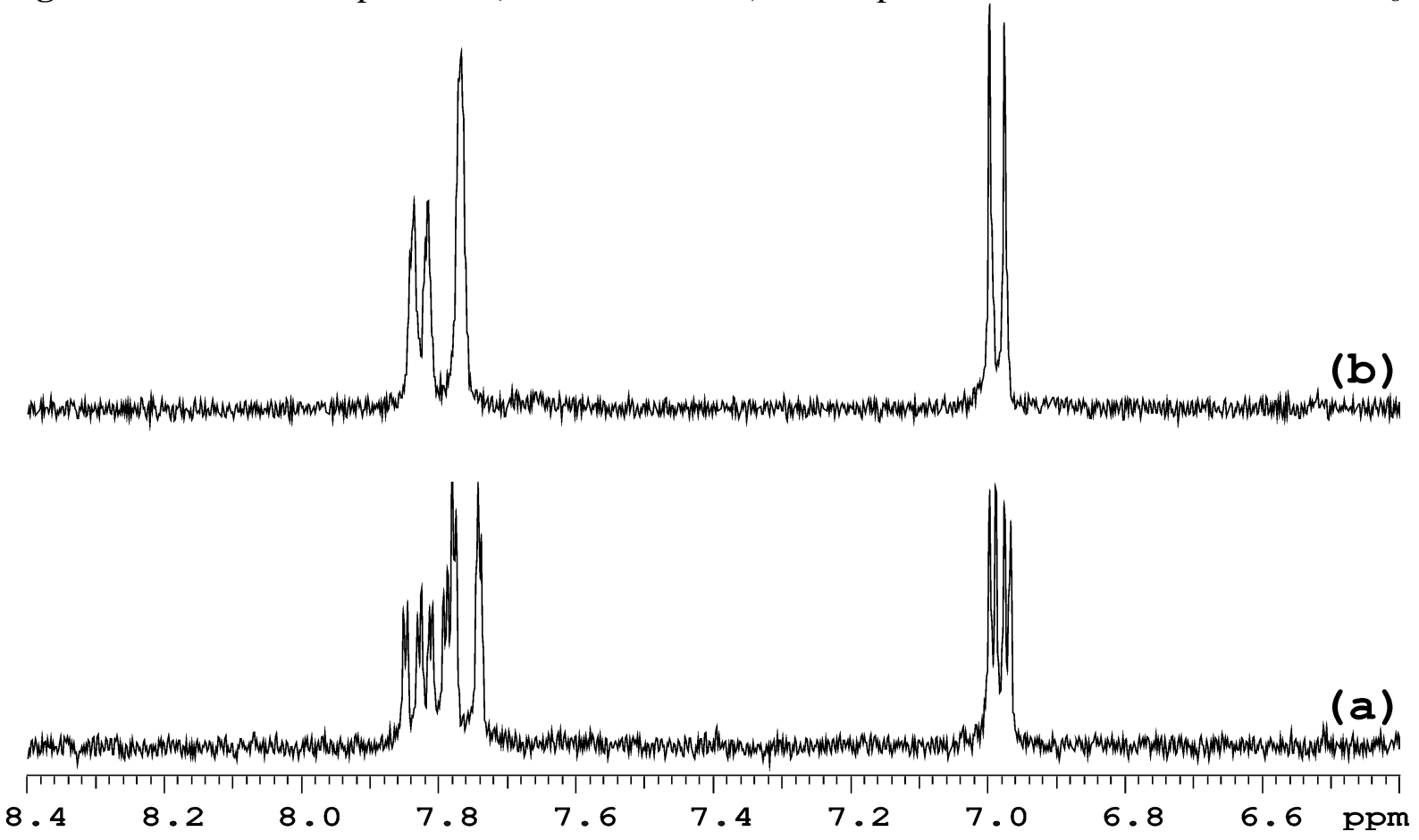
Figure S19. Aromatic region of the ${ }^{1} \mathrm{H}-\mathrm{NMR}$ spectrum $\left(\mathrm{THF}-\mathrm{d}_{8}, 25{ }^{\circ} \mathrm{C}, 400 \mathrm{MHz}\right.$ ) of compound 8, (a) without ${ }^{31} \mathrm{P}$ decoupling and (b) with ${ }^{31} \mathrm{P}$ bandselective adiabatic heteronuclear decoupling (WURST). Experiment (b) has clarified the unambiguous assinment of the spin system.

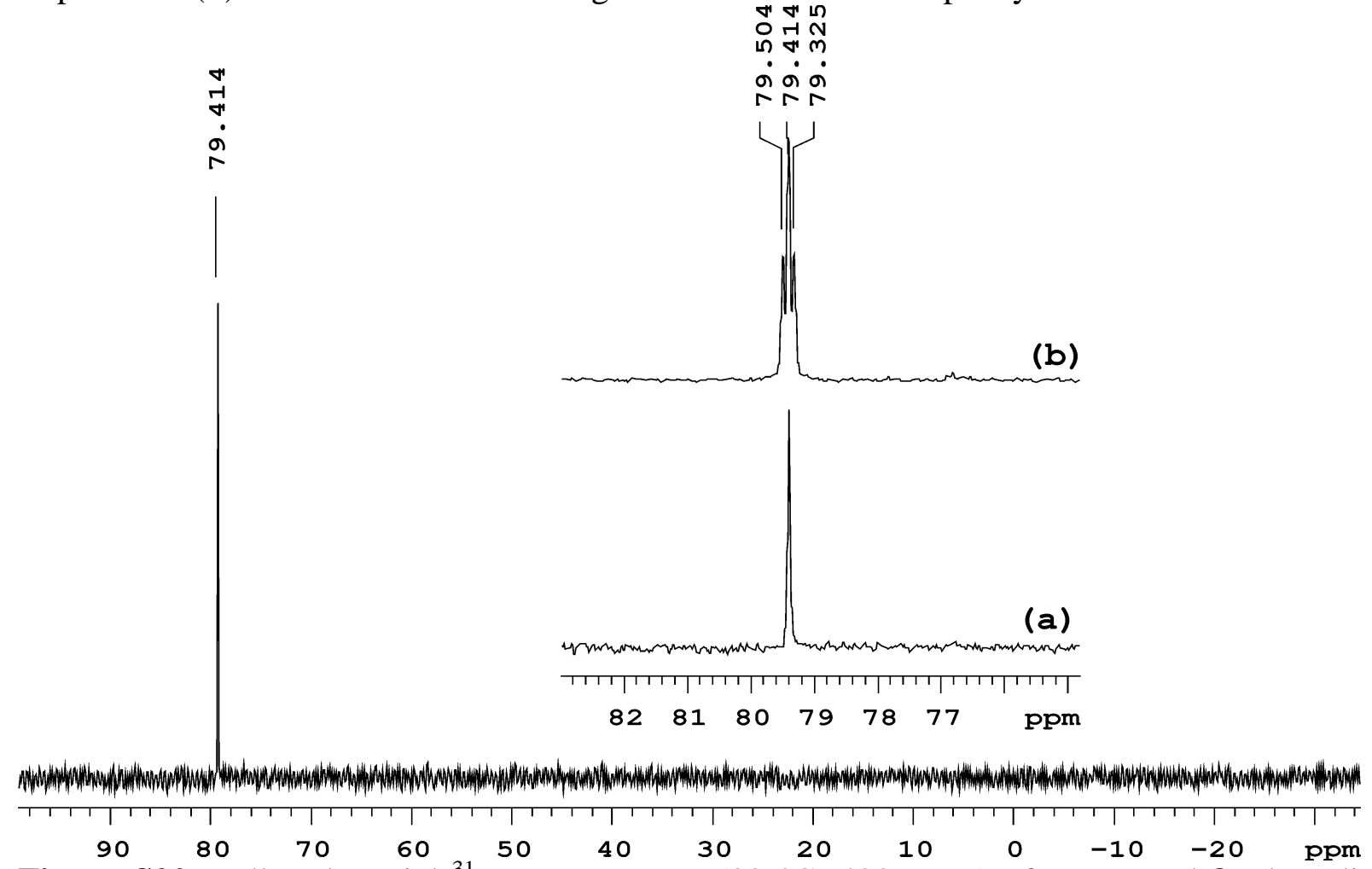

Figure S20. Full and partial ${ }^{31} \mathrm{P}-\mathrm{NMR}$ spectra $\left(30{ }^{\circ} \mathrm{C}, 400 \mathrm{MHz}\right)$ of compound 8 when dissolved in THF- $\mathrm{d}_{8},(\mathbf{a})$ and (b) are magnified regions with and without ${ }^{1} \mathrm{H}$-decoupling respectively. 


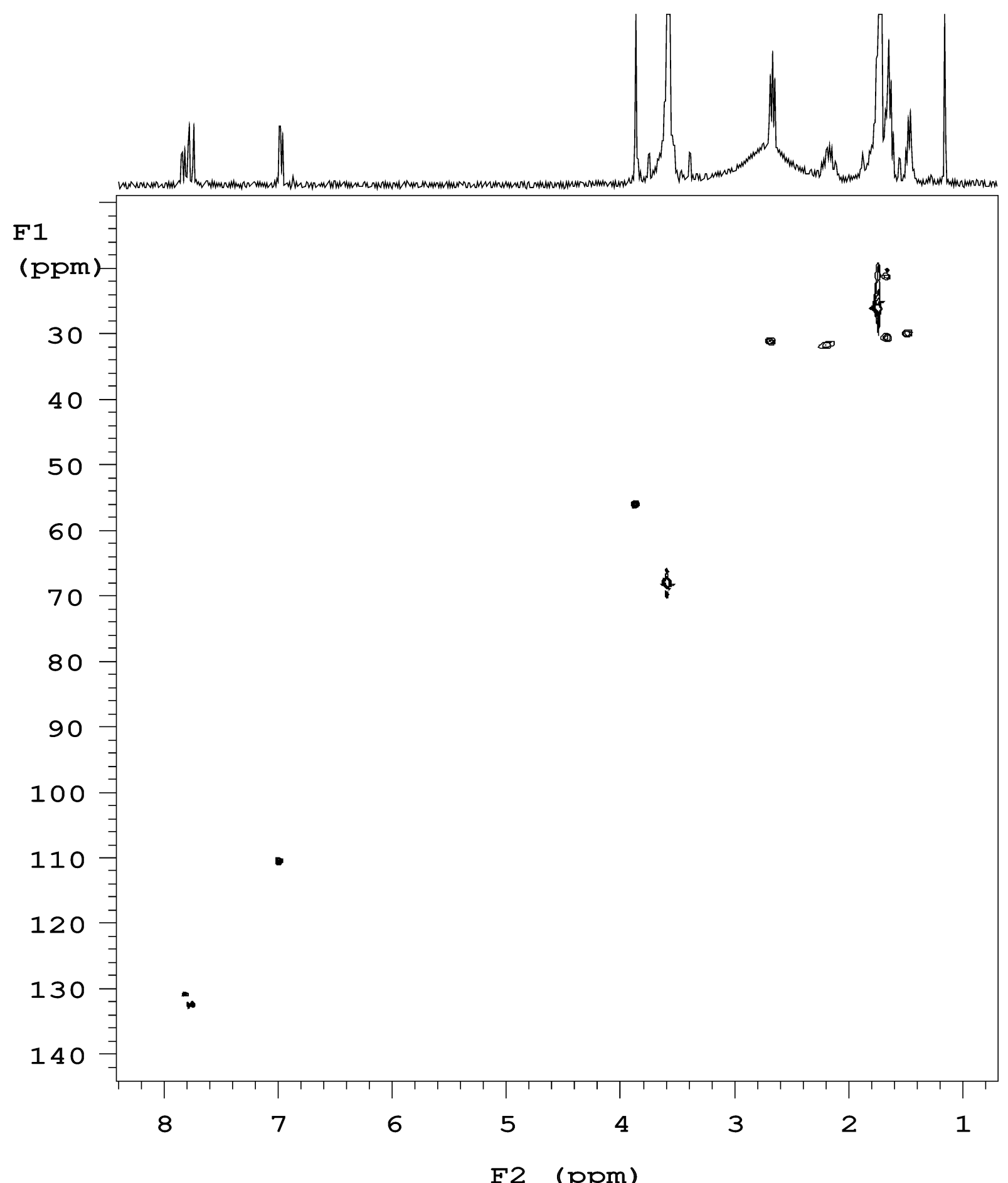

Figure S21. ${ }^{1} \mathrm{H}-{ }^{13} \mathrm{C}-\mathrm{gHSQC}$ spectrum $\left(\mathrm{THF}-\mathrm{d}_{8}, 25{ }^{\circ} \mathrm{C}, 400 \mathrm{MHz}\right.$ ) of compound 8. Direct $\mathrm{C}-\mathrm{H}$ correlations are shown as crosspeaks. The horizontal and vertical scales represent the ${ }^{1} \mathrm{H}$ and ${ }^{13} \mathrm{C}$ chemical shift dimensions respectively. 


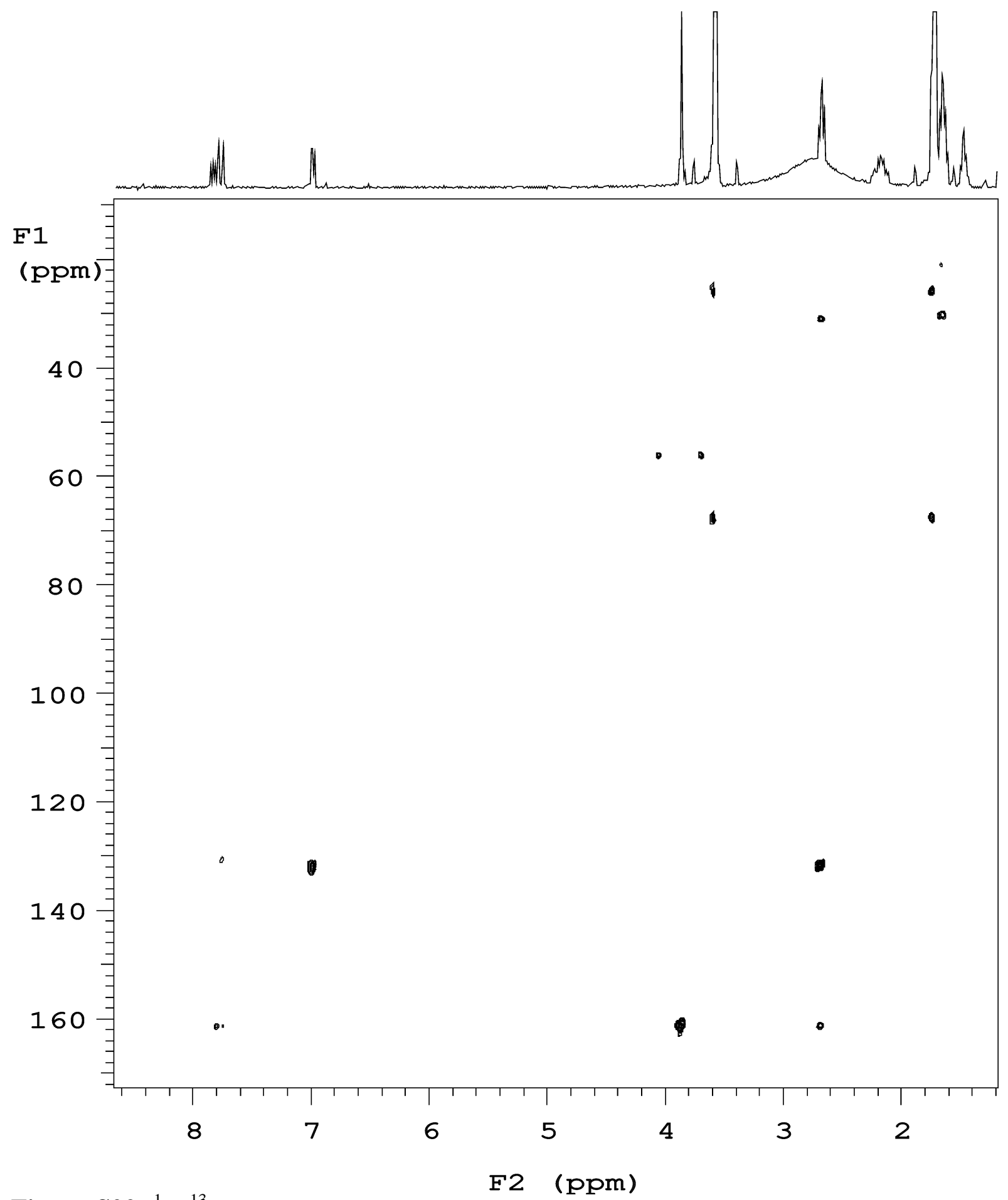

Figure S22. ${ }^{1} \mathrm{H}-{ }^{13} \mathrm{C}$-gHMQC spectrum $\left(\mathrm{THF}^{\mathrm{g}}-\mathrm{d}_{8}, 25^{\circ} \mathrm{C}, 400 \mathrm{MHz}\right.$ ) of compound 8. Three bond $\mathrm{C}-\mathrm{H}$ correlations ( $8 \mathrm{~Hz}$ optimized) are shown as crosspeaks. The horizontal and vertical scales represent the ${ }^{1} \mathrm{H}$ and ${ }^{13} \mathrm{C}$ chemical shift dimensions respectively. 


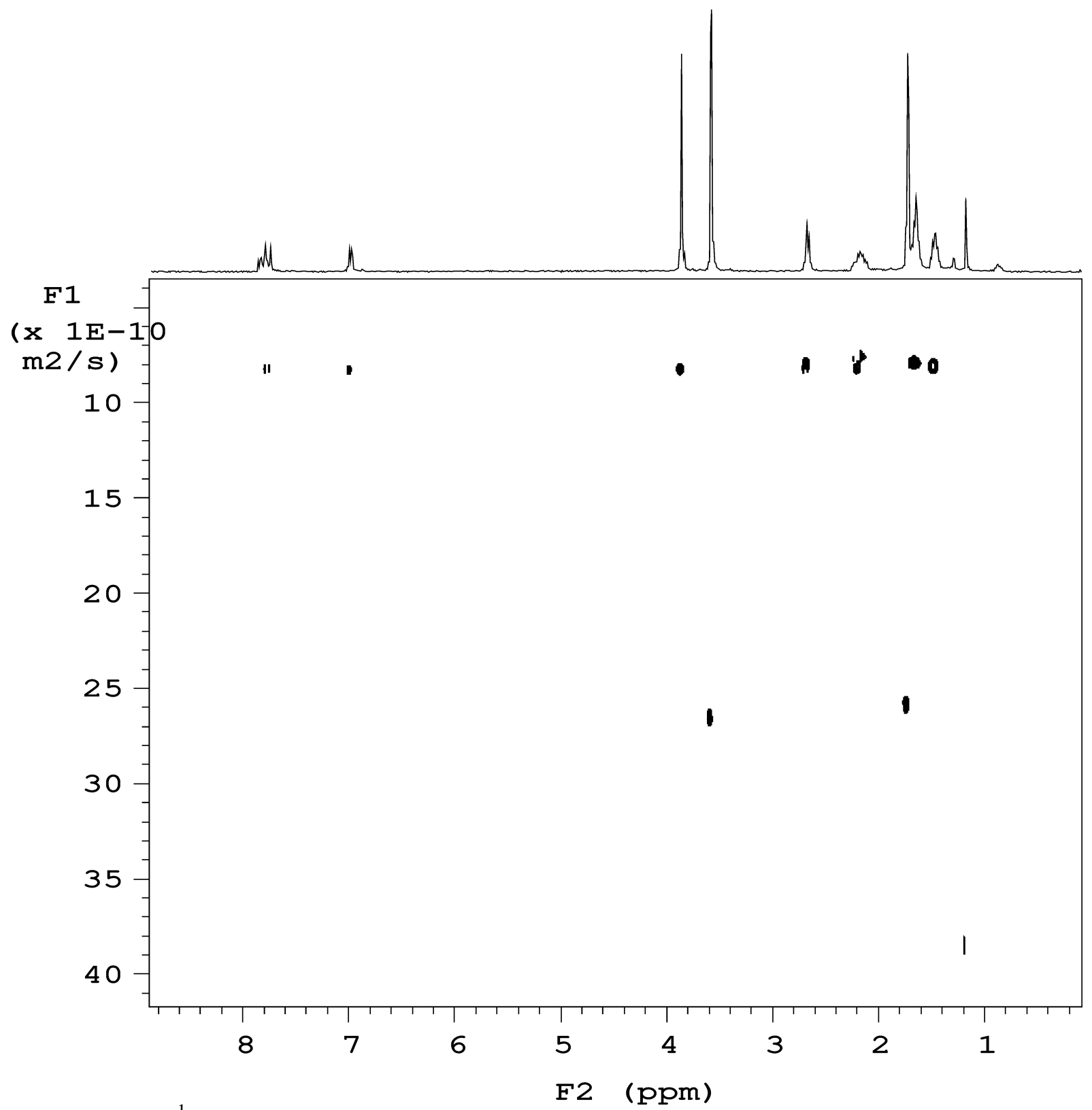

Figure S23. ${ }^{1} \mathrm{H}-\mathrm{DOSY}$ spectrum $\left(\mathrm{THF}-\mathrm{d}_{8}, 25{ }^{\circ} \mathrm{C}, 400 \mathrm{MHz}\right)$ of compound 8. Horizontal scale represents the ${ }^{1} \mathrm{H}$ chemical shift dimension whereas the vertical scale shows the diffusion dimension ( $\mathrm{x}$ $\left.10^{-10} \mathrm{~m}^{2} / \mathrm{s}\right)$ 


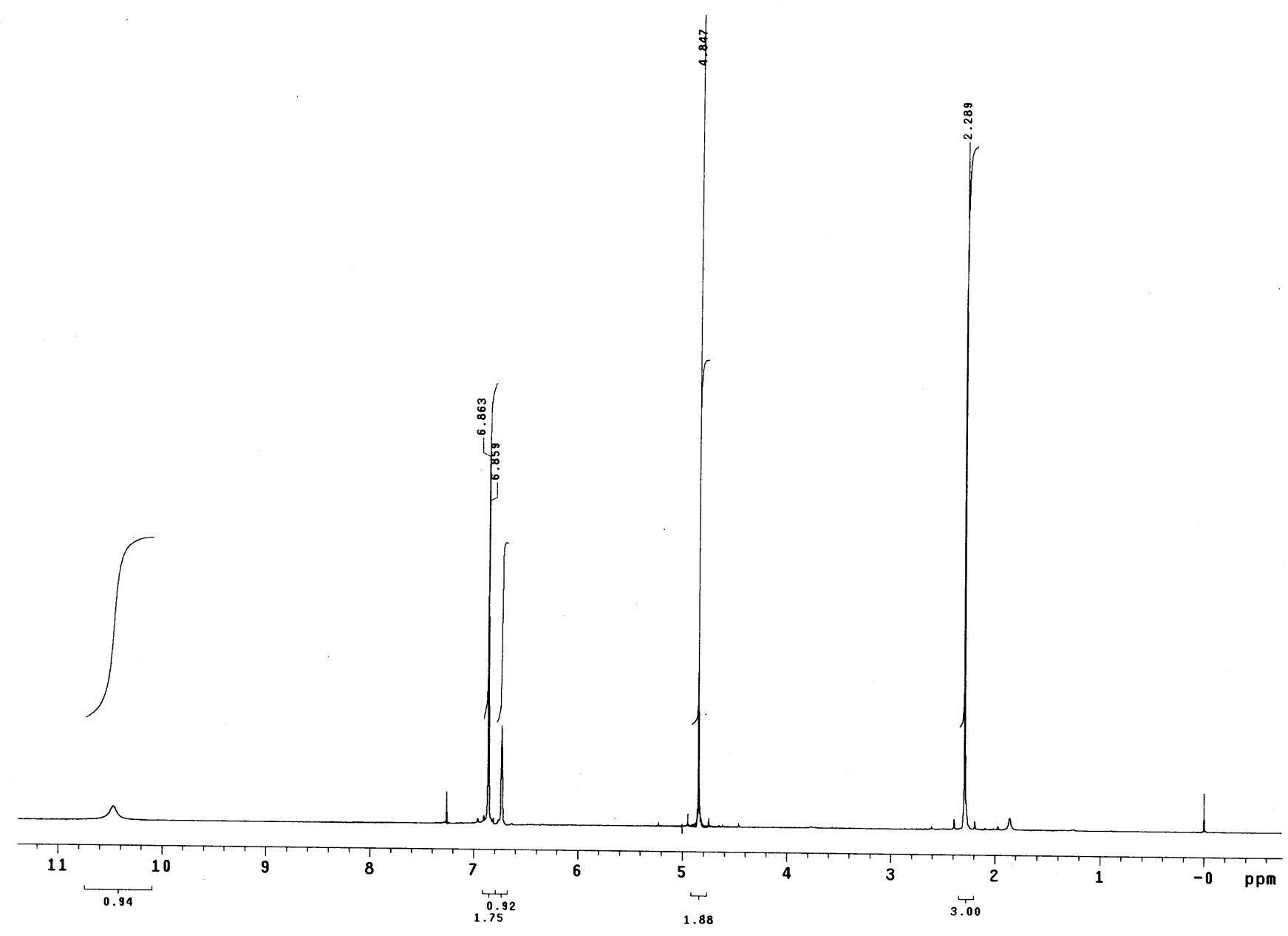

Figure S24. ${ }^{1} \mathrm{H}$ NMR spectra of compound $10 f$

\section{References:}

1 (a) Kupce, E; Freeman, R. J. Magn. Reson. 1995, 115, 273-276. (b) Kupce, E; Freeman, R. Chem. Phys. Lett. 1996, 250, 523-527.

2 Rocaboy, C.; Rutherford, D.; Bennett, B. L.; Gladysz, J. A. J. Phys. Org. Chem. 2000, 596-603.

3 Scheibye, S.; Pedersen, B. S.; Lawesson, S.-O. Bull. Soc. Chim. Belg. 1978, 87, 229-238.

4 Jensen, K. A.; Nielsen, P.H.; Acta Chem. Scand.; 1966, 20; 597-629.

5 Smith, D. C.; Lee, S. W.; Fuchs, P. L. J. Org. Chem. 1994, 59; 348-354.

6 Scheibye, S.; Kristensen, J.; Lawesson, S.-O. Tetrahedron 1979, 35, 1339-1343.

7 Aitken, R. A.; Drysdale, M. J.; Ryan, B. M. J. Chem. Soc., Perkin Trans. 1. 1998, 3345-3348.

8 Dodd, D. S.; Marinez, R. L. Tetrahedron Lett. 2004, 45; 4265-4267.

9 Ostroumov, I. G.; Tsilko, A. E.; Maretina, I. A.; Petrov, A. A. J.Org.Chem.USSR (Engl.Transl.) 1987 1467-1474; Zh. Org. Khim. 1987, 1635-1643.

10 Tsuge, O., Inaba A. Bull. Chem. Soc. Jpn. 1973, 46, 2221-2225. 